Originalien

Orthopäde $2021 \cdot 50: 312-325$

https://doi.org/10.1007/s00132-020-03940-6

Online publiziert: 14 . Juli 2020

(c) Der/die Autor(en) 2020

H. Mühlhofer ${ }^{1} \cdot$ N. Renz ${ }^{2} \cdot$ A. Zahar ${ }^{3} \cdot$ M. Lüdemann ${ }^{4} \cdot$ M. Rudert $^{4} \cdot$ R. Hube ${ }^{5}$.

L. Frommelt ${ }^{6} \cdot$ R. Ascherl ${ }^{7} \cdot$ C. Perka ${ }^{2} \cdot$ R. von Eisenhart-Rothe ${ }^{1}$

${ }^{1}$ Klinik und Poliklinik für Orthopädie und Sportorthopädie, Klinikum rechts der Isar, Technische Universität München, München, Deutschland

${ }^{2}$ Centrum für Muskuloskeletale Chirurgie, Campus Charité Mitte, Charite, Berlin, Deutschland

${ }^{3}$ Helios Klinikum Emil von Behring, Berlin, Deutschland

${ }^{4}$ Orthopädische Klinik, König-Ludwig-Haus, Würzburg, Deutschland

${ }^{5}$ Orthopädische Chirurgie München, OCM Klinik München, München, Deutschland

${ }^{6}$ Helios ENDO-Klinik Hamburg, Hamburg, Deutschland

${ }^{7}$ Kirchham, Deutschland

\title{
Diagnostik der periprothetischen Infektion
}

\section{Entwicklung eines evidenzbasierten Algorithmus der Arbeitsgruppe implantatassoziierte Infektion der Arbeitsgemeinschaft Endoprothetik}

und Low-Grade-Infektionen eingeteilt werden. Akute Infektionen werden durch hochvirulente Erreger, wie z. B. Staphylococcus aureus, hervorgerufen und treten entweder postoperativ durch direkte Kolonisation oder durch eine hämatogene Streuung auf. Sie sind oft durch klare Infektionszeichen wie Fieber und erhöhte laborchemische Entzündungsparameter gekennzeichnet. Als potenzieller Fokus für eine hämatogene Streuung kommen alle Arten von bakteriellen Infektionen im Körper infrage. Als klassische Vertreter sind hier kardiovaskuläre Infektionen (Endokarditis, Schrittmacherinfektion, Katheterinfektionen) bzw. Infektionen im Hals-Nasen-Ohren-Bereich, im Bereich der Zähne oder im Urogenitaltrakt zu nennen [67]. Low-Grade-Infektionen sind chronische Infekte, welche klinisch durch Prothesenlockerung und unspezifische Schmerzen im Bereich der Endoprothese bei Abwesenheit von akuten Entzündungszeichen auffallen. Diese werden oftmals durch niedrig virulente Erreger wie z. B. Staphylococcus epidermidis oder Cutibacterium (früher: Propionibacterium) acnes hervorgerufen [72]. Low-Grade-Infektionen werden am ehesten durch eine Kolonisation der Implantatkomponenten im Rahmen der Erstimplantation verursacht.

Der möglichst sichere Ausschluss oder die Bestätigung einer periprothetischen Infektion ist die Voraussetzung für jede Revisionsoperation und kann für den behandelnden Orthopäden und Unfallchirurgen eine große Herausforderung darstellen [89]. Eine sichere evidenzbasierte präoperative Diagnostik ist im Sinne des Patienten notwendig, um einerseits eine periprothetische Infektion $\mathrm{zu}$ erkennen sowie eine entsprechende chirurgische und antibiotische Therapie zu planen und andererseits, um unnötige zweizeitige Wechsel zu vermeiden. Gerade die Diagnostik von Low-Grade-Infektionen kann jedoch sehr herausfordernd sein. Neben der Anamnese und der klinischen Untersuchung stellen Röntgenaufnahmen, laborchemische Entzündungsparameter, Gelenkaspiration zur Bestimmung von Zellzahl und Zelldifferenzierung sowie mikrobiologischer Untersuchung der Synovialflüssigkeit bewährte diagnostische Routinen dar [87].

In den letzten Jahren stehen neu entwickelte Testverfahren, wie die moleku- 


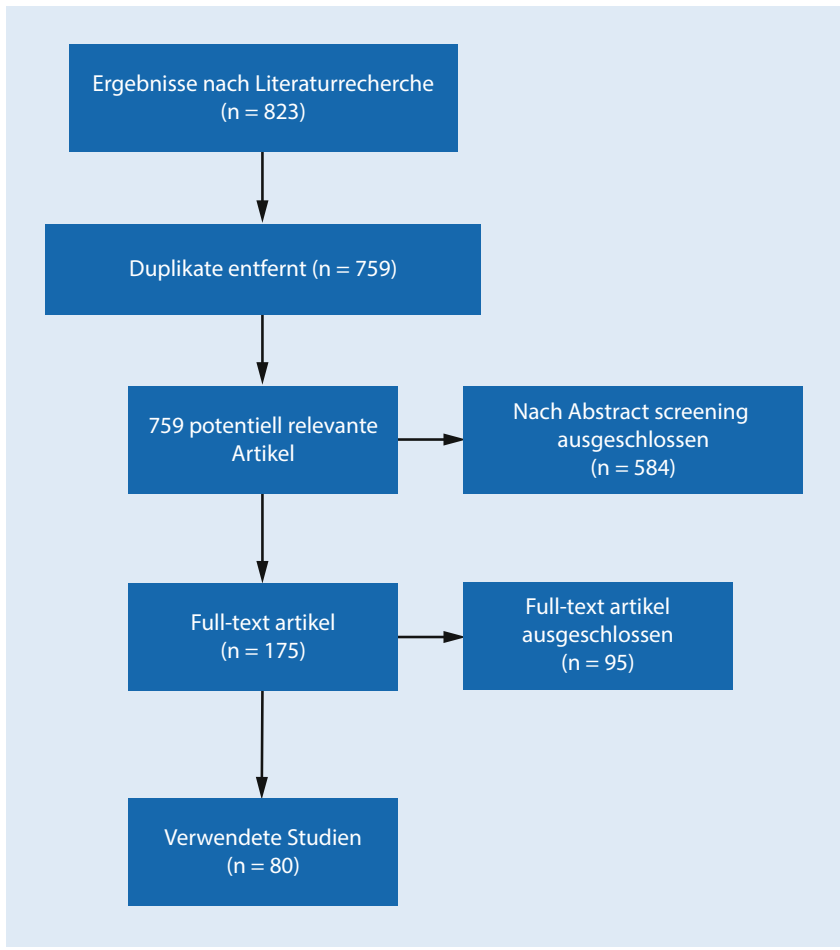

Abb. $1<$ Flow Chart der Studienauswahl larbiologische Diagnostik von Biomarkern (z. B. Alpha-Defensin), antimikrobiellen Peptiden (AMP) und der Leukozytenesterasetest zur Synovia-Diagnostik, sowie molekulargenetische Untersuchungen (16S/28S-PCR, Multiplex-PCR) zur Erregerbestimmung aus mikrobiologischen Biopsien zur Verfügung. Der Stellenwert dieser diagnostischen Möglichkeiten ist oftmals unklar und der behandelnde Orthopäde steht vor der großen Herausforderung, unter Berücksichtigung der zur Verfügung stehenden finanziellen Ressourcen des Gesundheitssystems die adäquate präoperative Diagnostik zu wählen.

Aus diesem Grund hat die Arbeitsgruppe für implantatassoziierte Infektionen ein evidenzbasiertes problem- und prioritätenbasiertes Vorgehen entwickelt und dies in einem transparenten und standardisierten Algorithmus zusammengefasst.

\section{Material und Methoden}

Im Rahmen der Treffen der Arbeitsgruppe für implantatassoziierte Infektionen der Arbeitsgemeinschaft Endoprothetik erfolgte eine systematische Literaturrecherche in Medline, Google Scholar und Web of Science mit folgenden Schlag- worten (Frage_ AND/OR): prosthetic joint infection, implant-associated infection, biofilm, diagnosis, sonication, antibiotic treatment, microcalorimetry, Staphylococcus aureus, coagulasenegative staphylococci, Propionibacterium, cutibacterium, rifampicin, implant retention, PCR, Maldi-TOF, serology, synovial fluid, C-reactive protein level, THA, TKA, leukocyte esterase test, alpha-defensin test. Alle relevanten Publikationen wurden hinsichtlich ihrer Methodik gemäß den Quadasund Prisma-Kriterien geprüft, bevor sie berücksichtigt wurden [51]. Alle eingeschlossenen Studien wurden gemäß den EAST-Kriterien bewertet und eingeteilt [28-34]. Insgesamt konnten 823 Publikationen identifiziert werden, die unserer Suchkriterien entsprochen haben (- Abb. 1). Weiterhin wurden vor allem Studien berücksichtigt, die eine international gängige Definitionen einer periprothetischen Infektion wie MSIS [59] (Musculoskeletal Infection Society) [76], IDSA (Infectious Diseases Society of America) [58], International Consensus Meeting [61] oder EBJIS (European Bone and Joint Infection Society) [68] angewandt haben. Insgesamt konnten so 80 Studien herangezogen werden. Aus diesen Studien wurden die entspre- chenden Daten extrahiert und folgende statistische Tests berechnet, falls dies noch nicht in der Originalpublikation erfolgt ist: Sensitivität, Spezifität, positive und negative Likelihood-Ratio, positive und negative prädiktive Werte.

Im Rahmen von 4 Treffen wurden die entsprechenden Studien der Arbeitsgruppe für implantatassoziierte Infektionen präsentiert und analog $\mathrm{zu}$ Standard-Delphi-Runden durch die einzelnen Experten bearbeitet und bewertet [69]. Gemäß der Prioritätenliste der Expertenrunde erfolgte die Entwicklung eines zur ISO (International Organization for Standardisation) konformen Algorithmus. Zur Anwendung kam die ISO-Norm 5807 (Informationsverarbeitung) modifiziert nach ITU-I, welche ursprünglich Dokumentationssymbole für Programm und Systemabläufe von Telekommunikationsprogrammnetzen bereitstellt. Durch die Modifikation erfolgte die Integration von DatenflussFlussdiagrammen (wie Algorithmen). So wird eine logische und standardisierte Entscheidungsfindung ermöglicht [39]. Hierzu werden verschiedenen Eingangsund Ausgangskriterien definiert, die von Prozess- und Entscheidungshexagons unterschieden werden. Durch Verwendung von Checklisten konnten die Entscheidungssymbole auf ein Minimum beschränkt werden. Grundsätzlich sollte eine DIN-A 4-Seite nicht überschritten werden.

\section{Ergebnisse der Arbeitsgruppe}

Der entwickelte Algorithmus ist eine Abfolge von evidenzbasierten Prozessen gemäß der verwendeten ISO-Norm. Gemäß der durch die Expertenrunde priorisierten Haupt- und Nebenkriterien erfolgte die Entwicklung logisch strukturiert und problemorientiert. Im horizontalen Flow werden Nebenkriterien dargestellt, im vertikalen Flow die Hauptkriterien. Checklisten wurden am linken Rand positioniert ([39]; • Abb. 2).

\section{Eingangskriterium}

Als Eingangskriterium, also Startpunkt des Algorithmus wurde von der Expertenrunde der „V.a. eine periprothetische 


\section{H. Mühlhofer · N. Renz · A. Zahar · M. Lüdemann · M. Rudert · R. Hube · L. Frommelt · R. Ascherl · C. Perka · R. von Eisenhart-Rothe Diagnostik der periprothetischen Infektion. Entwicklung eines evidenzbasierten Algorithmus der Arbeitsgruppe implantatassoziierte Infektion der Arbeitsgemeinschaft Endoprothetik}

\section{Zusammenfassung}

Hintergrund. Die Behandlung periprothetischer Infektionen (PPI) ist eine der größten Herausforderungen im Bereich der Endoprothetik. Der möglichst sichere Ausschluss oder die Bestätigung einer periprothetischen Infektion ist die Voraussetzung für jede Revisionsoperation und kann für den behandelnden Orthopäden und Unfallchirurgen eine große Herausforderung darstellen. Eine sichere evidenzbasierte präoperative Diagnostik ist im Sinne des Patienten notwendig, um einerseits eine periprothetische Infektion zu erkennen sowie die entsprechende chirurgische und antibiotische Therapie zu planen und andererseits unnötige zweizeitige Wechsel zu vermeiden.

Ziel der Arbeit. Ziel ist es, ein evidenzbasiertes problem- und prioritätenbasiertes Vorgehen zu entwickeln und dies in einem transparenten und standardisierten Algorithmus zusammenzufassen.
Methode. Durch systematische Literaturrecherche wurden relevante Arbeiten identifiziert und im Rahmen von Expertenrunden bewertet. Nach Extraktion der Daten erfolgte die Berechnung von Sensitivität, Spezifität, positiver und negativer LikelihoodRatio sowie positiver und negativer prädiktiver Werte. Im Rahmen von 4 Treffen wurden die entsprechenden Studien der Arbeitsgruppe für implantatassoziierte Infektionen präsentiert und analog zu Standard-Delphi-Runden durch die einzelnen Experten bearbeitet und bewertet. Gemäß der Prioritätenliste der Expertenrunde erfolgte die Entwicklung eines zur ISO (International Organization for Standardisation) konformen Algorithmus. Ergebnisse. Der entwickelte Algorithmus ist eine Abfolge von evidenzbasierten Prozessen gemäß der verwendeten ISONorm. Gemäß der durch die Expertenrunde priorisierten Haupt- und Nebenkriterien erfolgte die Entwicklung logisch strukturiert und problemorientiert.

Schlussfolgerung. Der Ausschluss einer periprothetischen Infektion ist von enormer Bedeutung vor einer Revisionsoperation und entscheidet in vielen Fällen über den Erfolg und die Invasivität der Operation. Die Diagnose "periprothetische Infektion" erfordert eine substanzielle Veränderung der therapeutischen Strategie. Der durch die Arbeitsgruppe entwickelte Algorithmus fasst Positionen aus der aktuellen Literatur und spezielle Expertenmeinungen zusammen, dies ermöglicht einen transparenten diagnostischen Ansatz im Sinne einer Standard Operation Procedure.

Schlüsselwörter

Biofilm · Gelenkprothese · Protheseninfektionen · Sensitivität und Spezifität · Chirurgische Diagnosetechniken

\section{Diagnosis of periprosthetic joint infection. Development of an evidence-based algorithm by the work group of implant-associated infection of the AE-(German Society for Arthroplasty)}

\section{Abstract}

Background. Prosthetic joint infections (PPI) will challenge orthopaedic surgeons and the health care system in the coming years. Evidence-based and reliable preoperative diagnostics are necessary for success in the field of revision arthroplasty. Especially the preoperative detection of PPI is important with respect to the treatment strategy. Aim. The aim of this study was to develop a detailed and structured standard operating procedure (SOP) to detect PPI preoperatively. Methods. A systematic literature research was performed and relevant articles identified. After extracting the data, statistical calculations of sensitivity, specificity, positive/negative predictive value and positive/negative likelihood ratio were performed. The results were discussed and evaluated in four meetings analogously to standard Delphi rounds by the workgroup of implant-associated infections of the German AE (Arbeitsgemeinschaft Endoprothetik). An algorithm for the diagnostic approach according to ISO 5807 was made.

Results. The standardized algorithm combines a sequence of evidence-based procedures with detailed and structured main and additional criteria to every critical step in the diagnostic approach.
Conclusion. The detection of PPI is of tremendous importance prior to revision arthroplasty and determines its success or failure. The diagnosis "prosthetic joint infection" requires a substantial change with respect to treatment concepts. The algorithm summarizes current literature and specialized expert opinions in a modern standardized format for a transparent diagnostic approach.

Keywords

Biofilm - Joint prosthesis - Prosthesis-related infections · Sensitivity and specificity · Surgical diagnostic technic
Infektion" angenommen. Insgesamt wurde 39 Studien mit 312.946 Patienten zu diesem Thema eingeschlossen.

Große Multizenterstudien konnten Risikofaktoren bzw. Patientengruppen mit erhöhtem Risiko einer periprothetischen Infektion identifizieren. Gerade die Lockerung der Implantatkomponenten, periartikuläre Ossifikationen, eine Bewegungseinschränkung des Gelenkes sowie
Schmerzen im Bereich der Endoprothese und lokale Entzündungszeichen sollten den Verdacht auf einen periprothetischen Low-Grade-Infekt lenken [43]. Die Risikofaktoren und Evidenzgrade der Studien sind ausführlich in • Tab. 1 dargestellt.

Es kann nicht empfohlen werden, bei jedem Patienten der zu einer Wechseloperation ansteht (z. B. Inlayverschleiß) unkritisch und ohne Risikoabwägung eine Gelenkpunktion durchzuführen. Einerseits darf man potenzielle Komplikation, wie z.B. iatrogene Infektionen, nicht unberücksichtigt lassen. Murray et al. zeigen in einer Level-I-Studie bis zu 5,1\% Komplikationen nach Hüftpunktionen (Nachblutungen, Hämatome, Nervenläsionen, Infektionen). Barrack et al. beschreiben eine deutlich nied- 


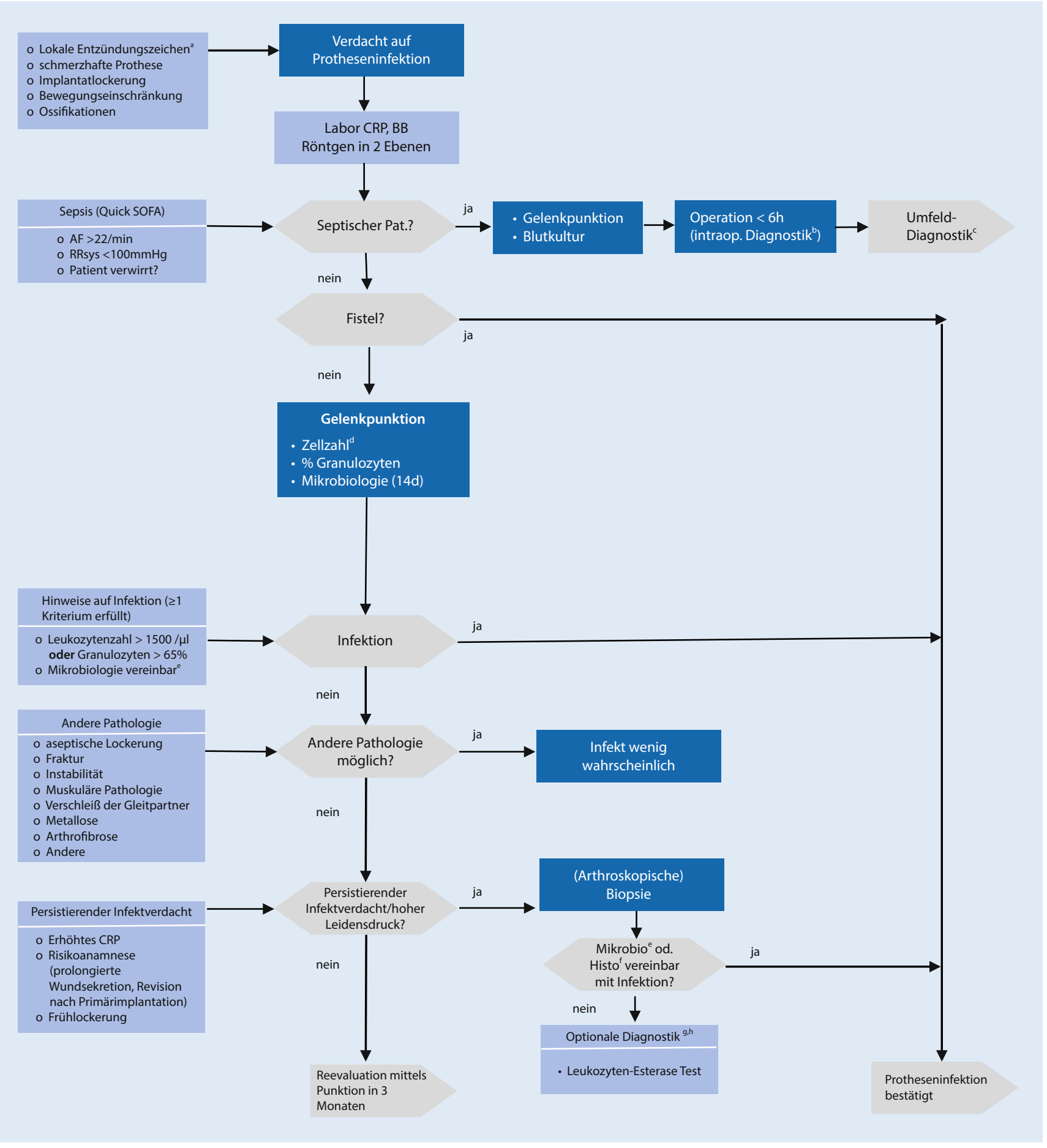

Abb. $2 \Delta$ Algorithmus zur Diagnostik periprothetischer Infektionen ${ }^{a}$ Beispiele für Symptome bei periprothetischem Infekt, Kriterien können keinesfalls alle möglichen Symptome abbilden; 'beukozytenzahl in Synovia, Anteil Granulozyten, Histopathologie, Mikrobiologie; 'Bildgebung Abdomen/Becken/ Wirbelsäule, transösophageale Echokardiografie, Orthopantomogramm, Urinanalyse, Thorax-Röntgen, (individuell bzw. abhängig vom Erreger und betroffenem Gelenk); ${ }^{d}$ bei rheumatischer Arthropathie, Fraktur und 6 Wochen postoperativ nicht zu verwenden; ${ }^{\mathrm{e}}$ bei hochvirulenten Erregern (S. aureus, E. coli) einmaliger Nachweis ausreichend, bei niedrig virulenten Erregern (S. epidermidis, Cutibacterium acnes) ist ein Nachweis in $\geq 2$ Proben notwendig; ${ }^{f} H i s t o l o-$ gie:Periprothetische Membran Typ II/III; ${ }^{9}$ ptionale peri-/intraoperative Diagnostik (Second Line) falls: a) keine elektive Diagnostik erfolgt ist z. B. aseptischer Wechsel mit intraoperativem Infektverdacht oder b) trotz Algorithmus unklarem Befund. Beachtung der Störanfälligkeit des Testes, kann aber individuell als hinweisend in Betracht gezogen werden. Leukozytenesterasetest: Cave Störanfällig durch Erythrozytenanteil; ggf. Zentrifugation zur Optimierung des Testes; ${ }^{\mathrm{h}}$ Testverfahren auf antimikrobielle Peptide (z. B. Alpha-Defensin) gewinnen zunehmend an Bedeutung, das Evidenzlevel der verfügbaren Studien ist aktuell noch nicht ausreichend, um Anwendungsempfehlung zu geben. Mit freundlicher Genehmigung, $\odot$ Arbeitsgemeinschaft Endoprothetik (AE), alle Rechte vorbehalten 
Tab. 1 Risikofaktoren für eine periprothetische Infektion, Darstellung der Evidenzlevel (LoE)

\begin{tabular}{|c|c|c|c|c|}
\hline Risikofaktor & Jahr & Autor & LoE & $(n=x)$ \\
\hline \multirow[t]{5}{*}{ Operationsdauer } & 2007 & Huotari et al. [35] & LoE II & $n=8201$ \\
\hline & 2004 & Småbrekke et al. [75] & LoE II & $n=31.750$ \\
\hline & 2010 & Kurtz et al. [42] & LoE II & $n=69.663$ \\
\hline & 2009 & Uçkay et al. [82] & LoE I & $n=6001$ \\
\hline & 1998 & Berbari et al. [7] & LoE II & $n=26.505$ \\
\hline \multirow[t]{9}{*}{ Übergewicht } & 2009 & Dowsey et al. [20] & LoE III & $n=1214$ \\
\hline & 2001 & Peersman et al. [63] & LoE III & $n=6120$ \\
\hline & 2007 & Lübbeke et al. [45] & LoE II & $n=2495$ \\
\hline & 2008 & Dowsey et al. [21] & LoE III & $n=1207$ \\
\hline & 2008 & Pulido et al. [66] & LoE III & $n=9245$ \\
\hline & 2012 & Namba et al. [56] & LoE II & $n=30.491$ \\
\hline & 2013 & Namba et al. [57] & LoE II & $n=56.216$ \\
\hline & 2009 & Malinzak et al. [48] & LoE III & $n=8494$ \\
\hline & 2011 & Peel et al. [62] & LoE III & $n=1200$ \\
\hline Mangelernährung & 2012 & Berbari et al. [8] & LoE III & $n=678$ \\
\hline \multirow[t]{4}{*}{ Diabetes } & 2013 & Namba et al. [57] & LoE II & $n=56.216$ \\
\hline & 2009 & Malinzak et al. [48] & LoE III & $n=8494$ \\
\hline & 2001 & Peersman et al. [63] & LoE III & $n=6120$ \\
\hline & 2011 & Mraovic et al. [52] & LoE III & $n=1948$ \\
\hline \multirow[t]{7}{*}{ Immunsuppression } & 2008 & Dowsey et al. [20] & LoE III & $n=1207$ \\
\hline & 2001 & Peersman et al. [63] & LoE III & $n=6120$ \\
\hline & 2009 & Jämsen et al. [36] & LoE II & $n=43.149$ \\
\hline & 2008 & Pulido et al. [66] & LoE II & $n=9245$ \\
\hline & 2011 & Peel et al. [62] & LoE III & $n=63$ \\
\hline & 1998 & Berbari et al. [7] & LoE II & $n=26.505$ \\
\hline & 2008 & Bongartz et al. [10] & LoE III & $n=462$ \\
\hline Vorausgegangener Infekt & 2009 & Jämsen et al. [36] & LoE II & $n=43.149$ \\
\hline \multirow[t]{3}{*}{ Bakteriämie } & 2010 & & LoE III & $n=126$ \\
\hline & 2013 & Coelho-Prabhu et al. [13] & LoE III & $n=678$ \\
\hline & 2001 & Murdoch et al. [54] & LoE III & $n=80$ \\
\hline \multirow[t]{2}{*}{ Infektion weiterer Prothesen } & 1991 & Murray et al. [55] & Level III & $n=159$ \\
\hline & 1996 & Luessenhop et al. [46] & Level III & $n=145$ \\
\hline Implantatlockerung & 2013 & Portillo et al. [65] & LoE I & $n=116$ \\
\hline Bakteriurie & 2013 & Sousa et al. [77] & LoE III & $n=2497$ \\
\hline Zahnstatus & 2010 & Berbari et al. [6] & Level III & $n=678$ \\
\hline Schmerzhafte Prothese & 2002 & Virolainen et al. [83] & Level III & $n=68$ \\
\hline Bewegungseinschränkung & 2013 & Parvizi et al. [61] & Level III & - \\
\hline Ossifikationen & 2002 & Breitenseher et al. [12] & Level III & - \\
\hline Lokale Entzündungszeichen & 2000 & Teller et al. [79] & Level III & $n=166$ \\
\hline
\end{tabular}

rigere Komplikationsrate von $1 \%$ [5]. Gemäß des Konsens der Expertengruppe stellt die Komplikationsrate durch die Punktion selbst nicht das vordergründige Problem dar. Die hohe Anzahl an Komplikationen in der Literatur kann so nicht nachvollzogen werden. Andererseits stellt ein weitaus größeres Problem die hohe Zahl an falsch positiven Be- weis eines Pathogens unterscheiden $\mathrm{zu}$ können, soll die Zellzahl in der Synovialflüssigkeit zu Hilfe genommen werden; erfolgt ein Erregernachweis bei völlig normaler Zellzahl, ist dieses Resultat mit Vorsicht zu interpretieren und eher als Kontamination $\mathrm{zu}$ werten. Im Hinblick auf die Synoviaaspiration bei liegender H-TEP muss auf die relativ hohe Anzahl an erfolglosen Punktionen (sogenannte Punctio sicca) hingewiesen werden. Diese treten überdurchschnittlich häufig bei aseptischen Hüften auf. Die Anzahl der falsch positiven Kulturen erhöht sich bei Punctio sicca von 7 auf 16,7\% [1] und die positive Likelihood-Ratio fällt von 8,9 auf 4,7. In diesem Zusammenhang muss darauf hingewiesen werden, dass auf eine Instillation mit Kochsalzlösung bei Punctio sicca verzichtet werden sollte.

Fazit Punktion Bei Vorliegen von Risikofaktoren für eine periprothetische Infektion (-Tab. 1; • Abb. 2) wird eine Punktion bzw. weitere Diagnostik empfohlen. Eine generelle, unkritische Punktion vor jeder Revisionsoperation kann nicht befürwortet werden.

\section{Checkliste Sepsis}

Als einer der ersten Schritte muss, um das zeitliche operative Vorgehen und die Dringlichkeit zu planen, eine Sepsis oder ein septischer Schock ausgeschlossen werden. Dies ist vor allem bei hochakuten periprothetischen Infektionen, welche oft durch eine hämatogene Streuung entstehen, relevant. Wir haben hierzu 10 Studien eingeschlossen, die sich mit dem Thema einer periprothetischen Infektion und Sepsis auseinandersetzen: $[18,44,74]$. Die Verwendung der SIRSKriterien wurde gegenüber der moderneren SOFA-Kriterien diskutiert. Als Konsensus erfolgte die Festlegung auf Quick-SOFA bei gleicher Evidenzklasse aufgrund der einfacheren Anwendung. Vor dem Beginn einer kalkulierten Antibiotikatherapie sollte eine Synoviaaspiration des betroffenen Gelenks erfolgen, um durch eine weitere mikrobiologische Untersuchung des Punktats den verursachenden Erreger zu identifizieren (Empfehlung Klasse III) Die Entnahme von Blutkulturen ist zwingend notwen- 
Tab. 2 Darstellung von Sensitivität, Spezifität, positiver/negativer Likelihood-Ratio (LR) sowie positiven und negativen prädiktiven Wert für laborchemische Parameter bei periprothetischen Infekt

\begin{tabular}{|c|c|c|c|c|c|c|c|c|c|c|c|}
\hline Jahr & Autor & $\begin{array}{l}\text { Unter- } \\
\text { suchung }\end{array}$ & LoE & CoR & $(n=x)$ & Sensitivität & Spezifität & Positive LR & $\begin{array}{l}\text { Negative } \\
\text { LR }\end{array}$ & $\begin{array}{l}\text { Positiv prädik- } \\
\text { tiver Wert }\end{array}$ & $\begin{array}{l}\text { Negativ } \\
\text { prädiktiver } \\
\text { Wert }\end{array}$ \\
\hline \multirow[t]{3}{*}{2007} & \multirow{3}{*}{$\begin{array}{l}\text { Bottner } \\
\text { et al. [11] }\end{array}$} & - & I & I & 78 & - & - & - & - & - & - \\
\hline & & $\begin{array}{l}\text { CRP } \\
(1,5 \mathrm{mg} / \mathrm{dl})\end{array}$ & - & - & - & $\begin{array}{l}0,95 \\
(0,86-1,0)\end{array}$ & $\begin{array}{l}0,91 \\
(0,94-0,99)\end{array}$ & $\begin{array}{l}10,86 \\
(5,3-73,07)\end{array}$ & $\begin{array}{l}0,05 \\
(0,0-0,17)\end{array}$ & $0,8(0,64-0,96)$ & $\begin{array}{l}0,98 \\
(0,94-1,0)\end{array}$ \\
\hline & & $\begin{array}{l}\text { ESR } \\
(32 \mathrm{~mm} / \mathrm{h})\end{array}$ & - & - & - & $\begin{array}{l}0,81 \\
(0,64-0,98)\end{array}$ & $\begin{array}{l}0,89 \\
(0,82-0,97)\end{array}$ & $\begin{array}{l}7,69 \\
(3,47-38,2)\end{array}$ & $\begin{array}{l}0,21 \\
(0,02-0,44)\end{array}$ & $\begin{array}{l}0,74 \\
(0,56-0,92)\end{array}$ & $\begin{array}{l}0,93 \\
(0,8-1,0)\end{array}$ \\
\hline \multirow[t]{3}{*}{2004} & \multirow{3}{*}{$\begin{array}{l}\text { Savarino } \\
\text { et al. [71] }\end{array}$} & - & I & I & 26 & - & - & - & - & - & - \\
\hline & & $\begin{array}{l}\text { ESR } \\
(50 \mathrm{~mm} / \mathrm{h})\end{array}$ & - & - & - & $\begin{array}{l}0,6 \\
(0,3-0,9)\end{array}$ & $\begin{array}{l}0,94 \\
(0,82-1,0)\end{array}$ & $\begin{array}{l}9,6 \\
(1,64-16,1)\end{array}$ & $\begin{array}{l}0,43 \\
(0,09-0,86)\end{array}$ & $0,86(0,71-1,0)$ & $\begin{array}{l}0,79 \\
(0,61-0,97)\end{array}$ \\
\hline & & $\begin{array}{l}\text { CRP } \\
(2 \mathrm{mg} / \mathrm{dl})\end{array}$ & - & - & - & $\begin{array}{l}0,38 \\
(0,14-0,61)\end{array}$ & $\begin{array}{l}0,7 \\
(0,42-0,98)\end{array}$ & $\begin{array}{l}1,25 \\
(0,24-38,34)\end{array}$ & $\begin{array}{l}0,89 \\
(0,39-2,07)\end{array}$ & $\begin{array}{l}0,67 \\
(0,36-0,97)\end{array}$ & $\begin{array}{l}0,42 \\
(0,18-0,65)\end{array}$ \\
\hline \multirow[t]{2}{*}{1980} & \multirow{2}{*}{$\begin{array}{l}\text { Kamme } \\
\text { et al. [37] }\end{array}$} & - & I & I & 63 & - & - & - & - & - & - \\
\hline & & $\begin{array}{l}\text { ESR } \\
(30 \mathrm{~mm} / \mathrm{h})\end{array}$ & - & - & - & $\begin{array}{l}0,89 \\
(0,8-0,99)\end{array}$ & $\begin{array}{l}0,72 \\
(0,54-0,9)\end{array}$ & $\begin{array}{l}3,2 \\
(1,75-9,54)\end{array}$ & $\begin{array}{l}0,15 \\
(0,01-0,37)\end{array}$ & $\begin{array}{l}0,83 \\
(0,71-0,94)\end{array}$ & $\begin{array}{l}0,82 \\
(0,66-0,98)\end{array}$ \\
\hline \multirow[t]{3}{*}{2007} & \multirow{3}{*}{$\begin{array}{l}\text { Greidanus } \\
\text { et al. [27] }\end{array}$} & - & I & I & 151 & - & - & - & - & - & - \\
\hline & & $\begin{array}{l}\text { ESR } \\
(30 \mathrm{~mm} / \mathrm{h})\end{array}$ & - & - & - & $\begin{array}{l}0,82 \\
(0,71-0,93)\end{array}$ & $\begin{array}{l}0,88 \\
(0,81-0,94)\end{array}$ & $\begin{array}{l}6,7 \\
(3,84-15,52)\end{array}$ & $\begin{array}{l}0,2 \\
(0,07-0,36)\end{array}$ & $\begin{array}{l}0,74 \\
(0,62-0,86)\end{array}$ & $\begin{array}{l}0,92 \\
(0,87-0,97)\end{array}$ \\
\hline & & $\begin{array}{l}\text { CRP } \\
(1,0 \mathrm{mg} / \mathrm{dl})\end{array}$ & - & - & - & $\begin{array}{l}0,93 \\
(0,86-1,0)\end{array}$ & $\begin{array}{l}0,83 \\
(0,76-0,9)\end{array}$ & $\begin{array}{l}5,5 \\
(3,57-10,23)\end{array}$ & $\begin{array}{l}0,08 \\
(0,01-0,18)\end{array}$ & $0,7(0,58-0,82)$ & $\begin{array}{l}0,97 \\
(0,93-1,0)\end{array}$ \\
\hline \multirow[t]{3}{*}{2007} & \multirow{3}{*}{$\begin{array}{l}\text { Della } \\
\text { Valle } \\
\text { et al. [17] }\end{array}$} & - & I & I & 94 & - & - & - & - & - & - \\
\hline & & $\begin{array}{l}\text { ESR } \\
(30 \mathrm{~mm} / \mathrm{h})\end{array}$ & - & - & - & $\begin{array}{l}0,9 \\
(0,81-0,99)\end{array}$ & $\begin{array}{l}0,66 \\
(0,53-0,79)\end{array}$ & $\begin{array}{l}2,66 \\
(1,74-4,68)\end{array}$ & $\begin{array}{l}0,15 \\
(0,01-0,35)\end{array}$ & $0,67(0,55-0,8)$ & $\begin{array}{l}0,9 \\
(0,8-0,99)\end{array}$ \\
\hline & & $\begin{array}{l}\text { CRP } \\
(1 \mathrm{mg} / \mathrm{dl})\end{array}$ & - & - & - & $\begin{array}{l}0,95 \\
(0,89-1,00)\end{array}$ & $\begin{array}{l}0,75 \\
(0,64-0,87)\end{array}$ & $\begin{array}{l}3,88 \\
(2,45-7,86)\end{array}$ & $\begin{array}{l}0,06 \\
(0,02-0,18)\end{array}$ & $\begin{array}{l}0,75 \\
(0,63-0,87)\end{array}$ & $\begin{array}{l}0,95 \\
(0,89-1,0)\end{array}$ \\
\hline \multirow[t]{3}{*}{2008} & \multirow{3}{*}{$\begin{array}{l}\text { Schinsky } \\
\text { et al. [73] }\end{array}$} & - & I & I & 201 & - & - & - & - & - & - \\
\hline & & $\begin{array}{l}\text { ESR } \\
(30 \mathrm{~mm} / \mathrm{h})\end{array}$ & - & - & - & $\begin{array}{l}0,96 \\
(0,91-1,0)\end{array}$ & $\begin{array}{l}0,39 \\
(0,31-0,47)\end{array}$ & $\begin{array}{l}1,58 \\
(1,33-1,91)\end{array}$ & $\begin{array}{l}0,09 \\
(0,0-0,28)\end{array}$ & $\begin{array}{l}0,37 \\
(0,29-0,45)\end{array}$ & $\begin{array}{l}0,97 \\
(0,92-1,0)\end{array}$ \\
\hline & & $\begin{array}{l}\text { CRP } \\
(1 \mathrm{mg} / \mathrm{dl})\end{array}$ & - & - & - & $\begin{array}{l}0,95 \\
(0,89-1,0)\end{array}$ & $\begin{array}{l}0,71 \\
(0,94-1,0)\end{array}$ & $\begin{array}{l}3,29 \\
(2,45-4,69)\end{array}$ & $\begin{array}{l}0,08 \\
(0,0-0,18)\end{array}$ & $\begin{array}{l}0,55 \\
(0,45-0,65)\end{array}$ & $\begin{array}{l}0,97 \\
(0,94-1,0)\end{array}$ \\
\hline \multirow[t]{2}{*}{2007} & \multirow{2}{*}{$\begin{array}{l}\text { Fink et al. } \\
{[24]}\end{array}$} & - & 1 & I & 145 & - & - & - & - & - & - \\
\hline & & $\begin{array}{l}\text { CRP } \\
(1,35 \mathrm{mg} / \mathrm{dl})\end{array}$ & - & - & - & $\begin{array}{l}0,73 \\
(0,59-0,86)\end{array}$ & $\begin{array}{l}0,81 \\
(0,73-0,88)\end{array}$ & $\begin{array}{l}3,81 \\
(2,21-7,48)\end{array}$ & $\begin{array}{l}0,34 \\
(0,15-0,56)\end{array}$ & $\begin{array}{l}0,59 \\
(0,45-0,73)\end{array}$ & $\begin{array}{l}0,89 \\
(0,82-0,95)\end{array}$ \\
\hline
\end{tabular}

CRP C-reaktives Protein, CoR Class of Recommendation (EAST), ESR Erythrozytensedimentationsrate, LoE Level of Evidence (EAST), EAST Eastern Association for the Surgery of Trauma

dig. Gemäß einer Klasse-II-Empfehlung senkt die zeitnahe chirurgische Versorgung die Mortalität.

Fazit Sepsis Aufgrund der hohen Mortalitätsrate besteht bei V.a. auf ein septisches Krankheitsbild umgehender interdisziplinärer Handlungsbedarf mit Entnahme von Blutkulturen, zeitnaher Antibiotikatherapie und chirurgischer Intervention im Sinne einer „source control“.

\section{Laborchemische Entzündungs- parameter}

Im Rahmen der vielzitieren AAOSRichtlinie wird zum Ausschluss einer pe- riprothetischen Infektion ein kompliziertes Zusammenspiel von laborchemischen Parametern (Blutsenkungsgeschwindigkeit/C-reaktives Protein) verwendet [60]. In dieser Metaanalyse werden Level-Iund Level-II-Studien berücksichtigt, die errechneten Sensitivitäten variieren von 81-93\% bezüglich der BSG sowie von 73-95\% für das C-reaktive Protein. Eine kürzlich veröffentlichte Metaanalyse von Berbari et al. zeigte ähnliche Ergebnisse. Bei einem Studienkollektiv von insgesamt 3909 Patienten errechnete sich eine gepoolte Sensitivität von $75 \%$ (BSG) und $88 \%$ (CRP) [6]. Hier muss jedoch berücksichtigt werden, dass die Studien mit den verwendeten Diagnosekriteri- en mehrheitlich deutliche Infektionen eingeschlossen haben und Low-GradeInfektionen - welche typischerweise tiefe systemische Entzündungswerte hervorrufen - mit diesen Kriterien größtenteils nicht erfasst wurden. Somit sind die Sensitivitäten vermutlich deutlich überschätzt. Insbesondere beim CRP zeigen sich unbefriedigende Werte im Bereich der Sensitivität. McArthur et al. bestätigen diese Ergebnisse in ihrem Kollektiv mit einer relevant großen Gruppe von Patienten mit einem periprothetischen Infekt ohne relevante serologische Entzündungszeichen [50]. Im Gegensatz hierzu empfehlen die AAOS-Guidelines eine Synoviaaspiration nur bei erhöhten 
Tab. 3 Darstellung von Sensitivität, Spezifität, positiver/negativer Likelihood-Ratio (LR) sowie positiven und negativen prädiktiven Wert für die Synoviaanalyse bei K-TEP

\begin{tabular}{|c|c|c|c|c|c|c|c|c|c|c|c|}
\hline Jahr & Autor & $\begin{array}{l}\text { Unter- } \\
\text { suchung }\end{array}$ & LoE & CoR & (n) & Sensitivität & Spezifität & Positive LR & $\begin{array}{l}\text { Negative } \\
\text { LR }\end{array}$ & $\begin{array}{l}\text { Positiv prädik- } \\
\text { tiver Wert }\end{array}$ & $\begin{array}{l}\text { Negativ prä- } \\
\text { diktiver Wert }\end{array}$ \\
\hline \multirow[t]{4}{*}{2007} & \multirow{4}{*}{$\begin{array}{l}\text { Della } \\
\text { Valle } \\
\text { et al. [17] }\end{array}$} & - & I & I & 94 & - & - & - & - & - & - \\
\hline & & $\begin{array}{l}\text { Mikro- } \\
\text { biologie }\end{array}$ & - & - & - & 0,8 & 0,93 & n.m. & n.m. & 0,94 & 0,84 \\
\hline & & $\begin{array}{l}\text { Zellzahl } \\
\left(3,0 \times 10^{3}\right)\end{array}$ & - & - & - & $\begin{array}{l}0,98 \\
(0,93-1,9)\end{array}$ & 1 & $x$ & 0,02 & 1 & $0,98(0,94-1,0)$ \\
\hline & & Zelldiff. & - & - & - & $\begin{array}{l}0,98 \\
(0,93-1,0)\end{array}$ & $\begin{array}{l}0,85 \\
(0,75-0,95)\end{array}$ & $\begin{array}{l}6,46 \\
(3,75-18,75)\end{array}$ & $0,03(0-0,1)$ & $\begin{array}{l}0,83 \\
(0,73-0,94)\end{array}$ & $0,98(0,94-1,0)$ \\
\hline \multirow[t]{2}{*}{2007} & \multirow{2}{*}{$\begin{array}{l}\text { Fink et al. } \\
\text { [24] }\end{array}$} & - & I & I & 145 & - & - & - & - & - & - \\
\hline & & $\begin{array}{l}\text { Mikro- } \\
\text { biologie }\end{array}$ & - & - & - & $\begin{array}{l}0,73 \\
(0,59-0,86)\end{array}$ & $\begin{array}{l}0,95 \\
(0,91-0,99)\end{array}$ & $\begin{array}{l}15,23 \\
(6,64-125,4)\end{array}$ & $\begin{array}{l}0,29 \\
(0,14-0,45)\end{array}$ & $\begin{array}{l}0,85 \\
(0,73-0,97)\end{array}$ & $0,9(0,85-0,96)$ \\
\hline \multirow[t]{3}{*}{2008} & \multirow{3}{*}{$\begin{array}{l}\text { Ghanem } \\
\text { et al. [26] }\end{array}$} & - & I & I & 429 & - & - & - & - & - & - \\
\hline & & Zellzahl & - & - & - & $\begin{array}{l}0,91 \\
(0,86-0,95)\end{array}$ & $\begin{array}{l}0,88 \\
(0,84-0,92)\end{array}$ & $\begin{array}{l}7,59 \\
(5,45-11,81)\end{array}$ & $\begin{array}{l}0,11 \\
(0,05-0,16)\end{array}$ & $\begin{array}{l}0,82 \\
(0,76-0,88)\end{array}$ & $\begin{array}{l}0,94 \\
(0,91-0,97)\end{array}$ \\
\hline & & Zelldiff. & - & - & - & $\begin{array}{l}0,95 \\
(0,92-0,98)\end{array}$ & $\begin{array}{l}0,95 \\
(0,92-0,97)\end{array}$ & $\begin{array}{l}18,19 \\
(11,62-38,43)\end{array}$ & $\begin{array}{l}0,05 \\
(0,02-0,09)\end{array}$ & $\begin{array}{l}0,92 \\
(0,87-0,96)\end{array}$ & $\begin{array}{l}0,97 \\
(0,95-0,99)\end{array}$ \\
\hline \multirow[t]{3}{*}{2007} & \multirow{3}{*}{$\begin{array}{l}\text { Trampuz } \\
\text { et al. [80] }\end{array}$} & - & I & 1 & - & - & - & - & - & - & - \\
\hline & & $\begin{array}{l}\text { Zellzahl } \\
\left(1,7 \times 10^{3}\right)\end{array}$ & - & - & - & $\begin{array}{l}0,94 \\
(0,86-1,0)\end{array}$ & $\begin{array}{l}0,88 \\
(0,81-0,94)\end{array}$ & $\begin{array}{l}7,76 \\
(4,65-17,92)\end{array}$ & $\begin{array}{l}0,07 \\
(0,0-0,17)\end{array}$ & $\begin{array}{l}0,73 \\
(0,60-0,86)\end{array}$ & $0,98(0,95-1,0)$ \\
\hline & & Zelldiff. & - & - & - & $\begin{array}{l}0,97 \\
(0,91-1,0)\end{array}$ & $\begin{array}{l}0,98 \\
(0,95-1,0)\end{array}$ & $\begin{array}{l}48,04 \\
(19,07-136,76)\end{array}$ & $\begin{array}{l}0,03 \\
(0,0-0,09)\end{array}$ & $0,94(0,87-1,0)$ & $0,99(0,97-1,0)$ \\
\hline \multirow[t]{3}{*}{2012} & \multirow{3}{*}{$\begin{array}{l}\text { Zmistow- } \\
\text { ski et al. } \\
{[88]}\end{array}$} & - & I & I & 150 & - & - & - & - & - & - \\
\hline & & $\begin{array}{l}\text { Zellzahl } \\
\left(3,0 \times 10^{3}\right)\end{array}$ & - & - & - & $\begin{array}{l}0,93 \\
(0,87-0,99)\end{array}$ & $\begin{array}{l}0,94 \\
(0,88-0,99)\end{array}$ & $\begin{array}{l}14,35 \\
(7,28-99)\end{array}$ & $\begin{array}{l}0,07 \\
(0,01-0,14)\end{array}$ & $\begin{array}{l}0,93 \\
(0,87-0,99)\end{array}$ & $\begin{array}{l}0,94 \\
(0,88-0,99)\end{array}$ \\
\hline & & Zelldiff. & - & - & - & $\begin{array}{l}0,93 \\
(0,87-0,99)\end{array}$ & $\begin{array}{l}0,83 \\
(0,75-0,91)\end{array}$ & $\begin{array}{l}5,52 \\
(3,46-11,62)\end{array}$ & $\begin{array}{l}0,08 \\
(0,01-0,17)\end{array}$ & $\begin{array}{l}0,84 \\
(0,76-0,92)\end{array}$ & $\begin{array}{l}0,93 \\
(0,87-0,99)\end{array}$ \\
\hline
\end{tabular}

Entzündungszeichen im Blut. Dieses Vorgehen würde die periprothetischen Infektionen ohne erhöhte systemischen Entzündungswerte von einer weiteren invasiven Diagnostik ausschließen. Die entsprechenden Studien sind in - Tab. 2 dargestellt. Die Arbeitsgruppe „implantatassoziierte Infektionen“ der Arbeitsgemeinschaft Endoprothetik hat in ihren Sitzungen die einzelnen Studien im Hinblick auf High- und Low-GradeInfekte bewertet. Diese Bewertung lässt den Schluss zu, dass, obwohl in den AAOS-Richtlinien Level-I-Studien als Basis der Empfehlung verwendet werden, in diesen zum Teil relativ alten Studien die Zahl der Low-Grade-Infekte sehr gering beziehungsweise teilweise nicht vorhanden ist. Gerade Low-Grade-Infekte sind durch Abwesenheit von lokalen und systemischen Infektparametern gekennzeichnet, da die auslösenden Erreger häufig niedrigvirulente Erreger, wie z. B.
Cutibacterium acnes oder Staphylococcus epidermidis sind.

Fazit Laborparameter Die Arbeitsgruppe sieht keine Möglichkeit zum sicheren Ausschluss bzw. der Bestätigung einer periprothetischen Infektion allein durch systemische Entzündungsparameter. Hierunter fallen auch neue moderne Entzündungsmarker wie Interleukine, Procalcitonin oder ähnliche. Die weitere Abklärung eines schmerzhaften Gelenkes sollte unabhängig von diesen Werten erfolgen.

\section{Fistel}

Gemäß MSIS [76], IDSA [58], International Consensus Meeting [61] und EBJIS [68] beweist das Vorhandensein einer Fistel, die mit einer Prothese kommuniziert, die periprothetische Infektion [9, 80].

\section{Goldstandard Synoviaanalyse}

Die Synoviaanalyse stellt den aktuellen Goldstandard in der präoperativen Diagnostik dar. Die aktuelle Literatur ist in - Tab. 3 und 4 dargestellt. Obwohl die Komplikationsraten nach Gelenkpunktion von künstlichen Knie- und Hüftgelenken als niedrig einzuschätzen sind, müssen Patienten vor diesem Eingriff formal aufgeklärt werden (siehe auch Eingangskriterium).

Die Analyse der Synoviaaspiration und die hieraus gewonnene mikrobiologische Kultur ist ein sehr kontrovers diskutiertes Thema in der Literatur. Die Kombination aus mikrobiologischer Langzeitbebrütung, Zellzahlbestimmung und Zelldifferenzierung entspricht dem aktuellen Goldstandard zur Analyse von Synoviaflüssigkeit. Die Zellzahl und Zelldifferenzierung sind äußerst wichtige Parameter der Synoviaanalyse. Einen entscheidenden Diskussionspunkt 
Tab. 4 Darstellung von Sensitivität, Spezifität, positiver/negativer Likelihood-Ratio (LR) sowie positiven und negativen prädiktiven Wert für die Synoviaanalyse bei H-TEP

\begin{tabular}{|c|c|c|c|c|c|c|c|c|c|c|c|}
\hline Jahr & Autor & $\begin{array}{l}\text { Unter- } \\
\text { suchung }\end{array}$ & LoE & CoR & $(n=x)$ & Sensitivität & Spezifität & Positive LR & $\begin{array}{l}\text { Negative } \\
\text { LR }\end{array}$ & $\begin{array}{l}\text { Positiv prä- } \\
\text { diktiver Wert }\end{array}$ & $\begin{array}{l}\text { Negativ prä- } \\
\text { diktiver Wert }\end{array}$ \\
\hline \multirow[t]{2}{*}{1993} & \multirow{2}{*}{$\begin{array}{l}\text { Barrack } \\
\text { et al. [4] }\end{array}$} & - & 1 & I & 291 & - & - & - & - & - & - \\
\hline & & $\begin{array}{l}\text { Mikro- } \\
\text { biologie }\end{array}$ & - & - & - & $0,6(0,3-0,9)$ & $\begin{array}{l}0,88 \\
(0,84-0,92)\end{array}$ & $\begin{array}{l}5,11 \\
(1,91-11,32)\end{array}$ & $\begin{array}{l}0,45 \\
(0,1-0,83)\end{array}$ & $\begin{array}{l}0,15 \\
(0,04-0,27)\end{array}$ & $\begin{array}{l}0,98 \\
(0,97-1,0)\end{array}$ \\
\hline \multirow[t]{2}{*}{1996} & \multirow{2}{*}{$\begin{array}{l}\text { Mulcahy } \\
\text { et al. [53] }\end{array}$} & - & 1 & I & 71 & - & - & - & - & - & - \\
\hline & & $\begin{array}{l}\text { Mikro- } \\
\text { biologie }\end{array}$ & - & - & - & $\begin{array}{l}0,69 \\
(0,46-0,91)\end{array}$ & $\begin{array}{l}0,91 \\
(0,83-0,99)\end{array}$ & $\begin{array}{l}7,56 \\
(2,76-61,25)\end{array}$ & $\begin{array}{l}0,34 \\
(0,09-0,65)\end{array}$ & $\begin{array}{l}0,69 \\
(0,46-0,91)\end{array}$ & $\begin{array}{l}0,91 \\
(0,83-0,99)\end{array}$ \\
\hline \multirow[t]{2}{*}{2004} & \multirow{2}{*}{$\begin{array}{l}\text { Williams } \\
\text { et al. [84] }\end{array}$} & - & 1 & I & 273 & - & - & - & - & - & - \\
\hline & & $\begin{array}{l}\text { Mikro- } \\
\text { biologie }\end{array}$ & - & - & - & $\begin{array}{l}0,8 \\
(0,71-0,9)\end{array}$ & $\begin{array}{l}0,94 \\
(0,9-0,97)\end{array}$ & $\begin{array}{l}12,47 \\
(7,23-29,34)\end{array}$ & $\begin{array}{l}0,21 \\
(0,11-0,32)\end{array}$ & $\begin{array}{l}0,81 \\
(0,72-0,91)\end{array}$ & $\begin{array}{l}0,93 \\
(0,90-0,97)\end{array}$ \\
\hline \multirow[t]{2}{*}{2004} & \multirow{2}{*}{$\begin{array}{l}\text { Malhotra } \\
\text { et al. [47] }\end{array}$} & - & 1 & I & 41 & - & - & - & - & - & - \\
\hline & & $\begin{array}{l}\text { Mikro- } \\
\text { biologie }\end{array}$ & - & - & - & $\begin{array}{l}0,44 \\
(0,12-0,77)\end{array}$ & $\begin{array}{l}0,91 \\
(0,81-1,0)\end{array}$ & $\begin{array}{l}4,74 \\
(0,62-106,19)\end{array}$ & $\begin{array}{l}0,61 \\
(0,23-1,09)\end{array}$ & $\begin{array}{l}0,57 \\
(0,2-0,94)\end{array}$ & $\begin{array}{l}0,85 \\
(0,73-0,97)\end{array}$ \\
\hline \multirow[t]{3}{*}{2008} & \multirow{3}{*}{$\begin{array}{l}\text { Schinsky } \\
\text { et al. [73] }\end{array}$} & - & 1 & I & 201 & - & - & - & - & - & - \\
\hline & & $\begin{array}{l}\text { Zellzahl } \\
\left(4,2 \times 10^{3}\right)\end{array}$ & - & - & - & $\begin{array}{l}0,84 \\
(0,74-0,93)\end{array}$ & $\begin{array}{l}0,93 \\
(0,89-0,97)\end{array}$ & $\begin{array}{l}12,21 \\
(6,75-33,94)\end{array}$ & $\begin{array}{l}0,18 \\
(0,07-0,29)\end{array}$ & $\begin{array}{l}0,82 \\
(0,72-0,92)\end{array}$ & $\begin{array}{l}0,94 \\
(0,90-0,98)\end{array}$ \\
\hline & & $\begin{array}{l}\text { Zelldiff. } \\
(80 \%)\end{array}$ & - & - & - & $\begin{array}{l}0,82 \\
(0,72-0,92)\end{array}$ & $\begin{array}{l}0,83 \\
(0,77-0,89)\end{array}$ & $\begin{array}{l}4,78 \\
(3,08-8,36)\end{array}$ & $\begin{array}{l}0,22 \\
(0,09-0,37)\end{array}$ & $\begin{array}{l}0,64 \\
(0,53-0,76)\end{array}$ & $\begin{array}{l}0,92 \\
(0,88-0,97)\end{array}$ \\
\hline \multirow[t]{3}{*}{1999} & \multirow{3}{*}{$\begin{array}{l}\text { Spangehl } \\
\text { et al. [78] }\end{array}$} & - & I & I & 183 & - & - & - & - & - & - \\
\hline & & $\begin{array}{l}\text { Zellzahl } \\
\left(5,0 \times 10^{3}\right)\end{array}$ & - & - & - & $\begin{array}{l}0,36 \\
(0,18-0,53)\end{array}$ & $\begin{array}{l}0,99 \\
(0,98-1,0)\end{array}$ & $\begin{array}{l}55,36 \\
(9,43-86,89)\end{array}$ & $\begin{array}{l}0,65 \\
(0,46-0,84)\end{array}$ & $\begin{array}{l}0,91 \\
(0,74-1,0)\end{array}$ & $\begin{array}{l}0,9 \\
(0,85-0,94)\end{array}$ \\
\hline & & $\begin{array}{l}\text { Zelldiff. } \\
(80 \%)\end{array}$ & - & - & - & $\begin{array}{l}0,89 \\
(0,78-1,0)\end{array}$ & $\begin{array}{l}0,85 \\
(079-0,91)\end{array}$ & $\begin{array}{l}5,94 \\
(3,76-10,75)\end{array}$ & $\begin{array}{l}0,13 \\
(0,0-0,28)\end{array}$ & $\begin{array}{l}0,52 \\
(0,38-0,66)\end{array}$ & $\begin{array}{l}0,98 \\
(0,95-1,0)\end{array}$ \\
\hline \multirow[t]{3}{*}{2013} & \multirow{3}{*}{$\begin{array}{l}\text { Dinneen } \\
\text { et al. [19] }\end{array}$} & - & 1 & I & 75 & - & - & - & - & - & - \\
\hline & & $\begin{array}{l}\text { Zellzahl } \\
\left(1,58 \times 10^{3}\right)\end{array}$ & - & - & - & $\begin{array}{l}0,895 \\
(0,783-0,997)\end{array}$ & $\begin{array}{l}0,913 \\
(0,827-0,999)\end{array}$ & - & - & - & - \\
\hline & & $\begin{array}{l}\text { Zelldiff. } \\
(65 \%)\end{array}$ & - & - & - & $\begin{array}{l}0,897 \\
(0,795-0,999)\end{array}$ & $\begin{array}{l}0,866 \\
(0,761-0,971)\end{array}$ & - & - & - & - \\
\hline
\end{tabular}

stellen die verwendeten Grenzwerte der Zellzahl und Zelldifferenzierung dar. Verschiedene Studien haben sich mit Grenzwertberechnungen von Zellzahlund Zelldifferenzierungsuntersuchungen auseinandergesetzt. Trampuz et al. schlagen $1,7 \times 10^{3} / \mu \mathrm{l}$ (Zellzahl) und $65 \%$ (Anteil neutrophile Granulozyten, Zelldifferenzierung) vor, Zmistowski et al. und Della Valle beschreiben vergleichbare Ergebnisse, obwohl ein höherer Grenzwert von $3,0 \times 10^{3} / \mu \mathrm{l}$ und $75 \%$ gewählt wurde. Die Grenzwertbestimmung erfolgten mittels ROC(„receiver-operating characteristics")-Kurven, diese Technik ist im Speziellen davon abhängig, welche Bakterien für die PPI in der ausgewählten Kohorte verantwortlich waren. Als Beispiel ist die Studie von Barrack et al. zu nennen, hier wurden als optimale Grenzwerte $4,2 \times 103 \mu$ l bzw. $75 \%$ verwendet. Das Kollektiv bestand aber überwiegend aus Patienten mit High-Grade-Infektionen und hochvirulenten Bakterien [73]. Die Expertengruppe hat sich aus diesem Grund auf einen niedrigeren Grenzwert im Algorithmus geeinigt, um auch chronische bzw. Low-Grade-Infektionen zu erfassen. Die Frage, ob Zellzahl und Zelldifferenzierung über den Grenzwerten liegen müssen, um von einer periprothetischen Infektion auszugehen, wurde ausführlich diskutiert. Als Konsens wurde festgelegt, dass entweder eine erhöhte Zellzahl oder eine erhöhte Zelldifferenzierung ausreicht, um eine Infektion wahrscheinlich zu machen.

Ein Problem der mikrobiologischen Kultur wird deutlich, wenn man die Literatur bezüglich der Sensitivitäten vergleicht. Hier zeigen sich sehr heterogene Daten: Der verursachende Erreger konnte in $44-80 \%$ der Fälle durch eine mikrobiologische Kultur nachgewiesen werden
[47, 84]. Ein entscheidender Faktor, der die Nachweiswahrscheinlichkeit deutlich erhöht, ist die Kulturdauer. Viele Studien mit geringer Sensitivität und Spezifität haben eine verkürzte Bebrütungszeit von $48 \mathrm{~h}$ angewendet bzw. die Kulturdauer nicht angegeben. Neben einer Bebrütungszeit von 14 Tagen ist die Antibiotikafreiheit bzw. das Absetzen einer bestehen Antibiotikatherapie mindestens 14 Tage vor Aspiration von Vorteil [70, 72]. Ein Problem sind falsch positive mikrobiologische Befunde, die oft durch Kontamination der mikrobiologischen Kultur verursacht werden. Dies führt, falls nicht durch einen Experten evaluiert und erkannt, zu einer massiven Übertherapie [86]. Hier ist es wichtig, dass die mikrobiologischen Resultate mit Nachweis von möglichen Kontaminanten (z.B. S. epidermidis, C. acnes) stets 
mit der Zellzahl im Punktat korreliert werden.

Fazit Synoviaanalyse Die Synoviaanalyse stellt den aktuellen Goldstandard dar. Als Grenzwerte empfehlen wir eine Leukozytenzahl von $1500 / \mu \mathrm{l}$ bzw. einen Granulozytenanteil von $>65 \%$. Der Nachweis einer erhöhten Zellzahl oder einer erhöhten Zelldifferenzierung ist als Infektion $\mathrm{zu}$ werten. Eine Langzeitbebrütung der mikrobiologischen Kultur von 14 Tagen ist erforderlich. Bei Nachweis von potenziellen Kontaminanten muss stets die Zellzahl zur Interpretation herangezogen werden.

\section{Weitere Verfahren in der Synoviaanalyse}

Andere neuartige synoviale Marker, wie der Alpha-Defensin-Test, zeigen vielversprechende Ergebnisse, jedoch sind die Fallzahlen in der aktuellen Literatur niedrig. Grundsätzlich muss zwischen einem kommerziell angebotenen Schnelltest (Minuten, qualitativ) und einem über verschiedene Labore angebotenen ELISA-Test (Tage, quantitativ) unterschieden werden. Die meisten Veröffentlichungen beziehen sich dabei auf den quantitativen ELISA-Test. Ein Problem stellen die selektiven Patientenkollektive dar, in vielen Studien wurden Patienten mit rheumatoider Arthritis oder abriebbedingter Metallose ausgeschlossen [14-16]. Kasparek et al. errechneten für den Alpha-DefensinTest eine Sensitivität von $67 \%$ bei einer Spezifität von $93 \%$ [38]. Renz et al. errechneten eine Sensitivität von $84 \%$ unter Verwendung der MSIS-Kriterien, von $67 \%$ unter Verwendung der IDSA-Kriterien und $54 \%$ unter Verwendung der PRO-IMPLANT/EBJIS-Kriterien [68]. Diese hochaktuellen Studien zeigten die noch bestehenden Probleme dieser neuartigen Biomarkertests. Konsens der Expertengruppe ist daher, dass die Alpha-Defensin-Bestimmung zum aktuellen Zeitpunkt nicht flächendeckend empfohlen werden kann.

Der Leukozytenesterasetest detektiert Leukozyten durch das in Leukozyten enthaltenen Enzym Leukozytenesterase. Durch eine chemische Reaktion kommt es am Teststreifen zu einem Farbumschlag, hierdurch wird semiquantitativ ein indirekter Leukozytennachweis im Punktat geführt. In einer Metaanalyse wird eine gepoolte Sensitivität von $81 \%$ bei einer Spezifität von $97 \%$ angegeben [85]. Aufgrund der problemlosen Verfügbarkeit und des extrem niedrigen Preises ist dieser Test als Konsens für eine Second-Line-Diagnostik unter bestimmten Umständen anwendbar. Insbesondere ist der Test aufgrund der hohen Spezifität bei positivem Resultat wegweisend. Wichtig ist hier die Limitation der fehlenden Verwertbarkeit bei Blutbeimengung des Punktats sowie die falsch positiven Resultate bei Nichteinhalten des Zeitfensters zur Ablesung des Resultates zu vermerken.

Fazit neue Biomarker Der flächendeckende Einsatz der Alpha-Defensin-Bestimmung kann bisher nicht empfohlen werden. Der Leukozytenesterasetest ist als Second-Line-Diagnostik verwendbar falls a) keine elektive Diagnostik erfolgt ist (z. B. aseptischer Wechsel mit intraoperativen Infektverdacht) oder b) trotz Algorithmus ein unklarer Befund vorliegt. Ein positiver Leukozytenesterasetest kann individuell als hinweisend in Betracht gezogen werden, schließt jedoch eine periprothetische Infektion weder mit ausreichender Sicherheit aus, noch bestätigt er diese.

\section{Biopsie von periprothetischem Gewebe zur mikrobiologischen und histologischen Aufarbeitung}

Ein weiterer Schritt bei persistierendem Infektverdacht (z. B. beim Ausschluss anderer Pathologien, widersprüchlichen Ergebnissen in der Synoviaaspiration), welcher jedoch deutlich invasiver (und somit komplikationsreicher und kostenintensiver) ist, stellt die Synovialisbiopsie dar. Durch Entnahme von Biopsaten kann eine wie von Malhotra and Morgan in ihrem Kollektiv vorgeschlagene Kombination aus mikrobiologischer Aufarbeitung und histopathologischer Untersuchung durchgeführt werden. Die Autoren berichten von einer Sensitivität von $80 \%$ bei einer Spezifität von $100 \%$ durch eine synoviale Biopsie im Vergleich $\mathrm{zu}$ deutlich niedrigeren Werten bei der Synoviaaspiration. (44\%/91\%). Fink et al. zeigten an mikrobiologischen Biopsien deren Überlegenheit im Vergleich zur Kultur aus der Synovialflüssigkeit. In Ihrem Kollektiv erreichten sie Sensitivitäten von $100 \%$ (K-TEP) und $87 \%$ (H-TEP) bei einer Spezifität von $98 \%$ [22, 23]. Pohlig et al. zeigten, wie die Sensitivität und Spezifität durch Kombination einer arthroskopisch gewonnen Biopsie mit mikrobiologischer und histopathologischer Untersuchung gesteigert werden können. Durch diese Kombination konnte die höchste Testgüte erreicht werden [64]. Als etabliertes Verfahren zur histopathologischen Einteilung ist die Morawietz-und-KrennKlassifikation $\mathrm{zu}$ nennen: hierbei werden die Granulozyten pro Gesichtsfeld in der periprothetischen Membran gezählt [40]. Die in den USA zum Teil etablierte Schnellschnittdiagnostik spielt in Deutschland eine eher untergeordnete Rolle. Im Gegensatz zur mikrobiologischen Biopsie spielt der Entnahmeort bei histopathologischen Proben eine außerordentlich wichtige Rolle. Ziel ist es, dass Teile der sogenannten SLIM („synovia-like interface membrane") oder Neosynovialis prothesennah gewonnen werden [40]. Technisch gesehen erfolgt die Biopsie am Kniegelenk durch die Standardportale der Kniearthroskopie, am Hüftgelenk sind in leichter Hüftflexion ein hohes und ein tiefes anterolaterales Portal geeignet, wie sie üblicherweise zur Adressierung des peripheren Gelenkkompartiments verwendet werden. Es werden 6 Proben des periprothetischen Gewebes entnommen und davon 5 zur mikrobiologischen und eine zur histologischen Untersuchung eingeschickt [2, 49]. Trotz der hervorragenden Testgüte bei synovialen Biopsien ( $\bullet$ Tab. 5) sollte die Indikation streng gestellt werden. Ein persistierender Infektverdacht bei positiver Risikoanamnese, Frühlockerung oder persistierenden unklaren Infektparametern können dies rechtfertigen. Obwohl arthroskopische Operationen als kleine Eingriffe gelten, sind diese nicht risikolos. Potenzielle Risken sind neben der Verletzung von neurovaskulären Strukturen die postoperative 
Tab. 5 Darstellung von Sensitivität, Spezifität, positiver/negativer Likelihood-Ratio (LR) sowie positiven und negativen prädiktiven Wert für die histopathologische Untersuchung

\begin{tabular}{|c|c|c|c|c|c|c|c|c|c|c|c|}
\hline Jahr & Autor & Untersuchung & LoE & CoR & $(n=x)$ & Sensitivität & Spezifität & Positive LR & $\begin{array}{l}\text { Negative } \\
\text { LR }\end{array}$ & $\begin{array}{l}\text { Positiv prä- } \\
\text { diktiver Wert }\end{array}$ & $\begin{array}{l}\text { Negativ prä- } \\
\text { diktiver Wert }\end{array}$ \\
\hline \multirow[t]{3}{*}{2002} & \multirow{3}{*}{$\begin{array}{l}\text { Banit } \\
\text { et al. [3] }\end{array}$} & - & I & I & - & - & - & - & - & - & - \\
\hline & & $\begin{array}{l}\text { Schnellschnitt } \\
\text { (Hüfte) }\end{array}$ & - & - & 63 & $\begin{array}{l}0,45 \\
(0,16-0,75)\end{array}$ & $\begin{array}{l}0,92 \\
(0,85-1,0)\end{array}$ & $\begin{array}{l}5,91 \\
(1,07-166,5)\end{array}$ & $\begin{array}{l}0,59 \\
(0,25-0,99)\end{array}$ & $\begin{array}{l}0,56 \\
(0,23-0,88)\end{array}$ & $\begin{array}{l}0,89 \\
(0,81-0,97)\end{array}$ \\
\hline & & $\begin{array}{l}\text { Schnellschnitt } \\
\text { (Knie) }\end{array}$ & - & - & 55 & 1 & $\begin{array}{l}0,96 \\
(0,9-1,0)\end{array}$ & $\begin{array}{l}23,00 \\
(9,76-64,7)\end{array}$ & 0 & $0,82(0,59-1,0)$ & 1 \\
\hline \multirow[t]{3}{*}{2007} & \multirow{3}{*}{$\begin{array}{l}\text { Francés } \\
\text { Borrego } \\
\text { et al. } \\
{[25]}\end{array}$} & - & 1 & I & - & - & - & - & - & - & - \\
\hline & & $\begin{array}{l}\text { Schnellschnitt } \\
\text { (Hüfte) }\end{array}$ & - & - & 83 & $\begin{array}{l}0,5 \\
(0,15-0,85)\end{array}$ & 1 & - & $\begin{array}{l}0,5 \\
(0,15-0,85)\end{array}$ & 1 & $0,95(0,9-1,0)$ \\
\hline & & $\begin{array}{l}\text { Schnellschnitt } \\
\text { (Knie) }\end{array}$ & - & - & 63 & $\begin{array}{l}0,67 \\
(0,48-0,86)\end{array}$ & $\begin{array}{l}0,9 \\
(0,8-0,99)\end{array}$ & $\begin{array}{l}6,5 \\
(2,42-116,4)\end{array}$ & $\begin{array}{l}0,37 \\
(0,15-0,65)\end{array}$ & $0,8(0,62-0,98)$ & $0,81(0,7-0,93)$ \\
\hline \multirow[t]{2}{*}{2008} & \multirow{2}{*}{$\begin{array}{l}\text { Schinsky } \\
\text { et al. } \\
\text { [73] }\end{array}$} & - & I & I & 201 & - & - & - & - & - & - \\
\hline & & $\begin{array}{l}\text { Schnellschnitt } \\
\text { (Hüfte) }\end{array}$ & - & - & - & $\begin{array}{l}0,73 \\
(0,61-0,84)\end{array}$ & $\begin{array}{l}0,94 \\
(0,9-0,98)\end{array}$ & $\begin{array}{l}11,8 \\
(6,06-37,34)\end{array}$ & $\begin{array}{l}0,29 \\
(0,16-0,43)\end{array}$ & $\begin{array}{l}0,82 \\
(0,71-0,92)\end{array}$ & $0,9(0,85-0,95)$ \\
\hline \multirow[t]{2}{*}{2008} & \multirow{2}{*}{$\begin{array}{l}\text { Fink } \\
\text { et al. } \\
\text { [23] }\end{array}$} & - & I & I & 145 & - & - & - & - & - & - \\
\hline & & Fixation & - & - & - & 1 & $\begin{array}{l}0,98 \\
(0,95-1,0)\end{array}$ & $\begin{array}{l}52,5 \\
(22,13-140,88)\end{array}$ & $x$ & $0,95(0,89-1,0)$ & 1 \\
\hline \multirow[t]{2}{*}{2007} & \multirow{2}{*}{$\begin{array}{l}\text { Della } \\
\text { Valle } \\
\text { et al. } \\
\text { [17] }\end{array}$} & - & I & I & 94 & - & - & - & - & - & - \\
\hline & & $\begin{array}{l}\text { Schnellschnitt } \\
\text { (Knie) }\end{array}$ & - & - & - & $\begin{array}{l}0,88 \\
(0,78-0,98)\end{array}$ & $\begin{array}{l}0,96 \\
(0,91-1,0)\end{array}$ & $\begin{array}{l}23,27 \\
(8,74-72,1)\end{array}$ & $\begin{array}{l}0,13 \\
(0,02-0,24)\end{array}$ & $0,95(0,88-1,0)$ & $\begin{array}{l}0,91 \\
(0,84-0,99)\end{array}$ \\
\hline \multirow[t]{2}{*}{2013} & \multirow{2}{*}{$\begin{array}{l}\text { Fink } \\
\text { et al. } \\
\text { [22] }\end{array}$} & - & I & I & 100 & - & - & - & - & - & - \\
\hline & & Fixation (Knie) & - & - & - & $\begin{array}{l}0,62 \\
(0,48-0,76)\end{array}$ & 1 & - & $\begin{array}{l}0,38 \\
(0,24-0,52)\end{array}$ & 1 & $\begin{array}{l}0,76 \\
(0,67-0,86)\end{array}$ \\
\hline
\end{tabular}

Infektion sowie die Beschädigung von Prothesenkomponenten durch die Arthroskopieinstrumente. Zudem ist das Risiko eines „Sampling-Errors“ durch Nichterreichen des repräsentativen Gewebes und konsekutiven falsch negativen Ergebnissen zu berücksichtigen. Wie auch die Synoviaaspiration stellt die Indikation zur arthroskopischen Biopsie eine Risiko-Nutzen-Abwägung dar, in der ein maximaler diagnostischer Gewinn bei minimalem Patientenrisiko erzeugt werden soll.

Fazit Biopsie Bei Diskrepanz zwischen Infektverdacht und unklaren Punktionsergebnissen sollte nach Ausschluss anderer Pathologien periprothetisches Gewebe gewonnen werden. Durch Kombination von mikrobiologischer bzw. histopathologischer Untersuchung der Biopsien kann die höchste diagnostische Güte erreicht werden - vorausgesetzt die Proben sind repräsentativ.

Die präoperativ vorliegenden Resultate müssen stets mit den im Rahmen der
Revisionsoperation entnommenen Proben korreliert und die Diagnose nach Vorliegen dieser erneut kritisch analysiert werden. Die intraoperativ entnommenen Biopsien sind oft aussagekräftiger, da das repräsentative Gewebe (z.B. Interface zwischen Prothese und Knochen) erreicht wird. Zudem kann die explantierte Prothese zur Sonikation geschickt werden, welche sich vor allem bei Low-Grade-Infektionen als sensitiver als Gewebeuntersuchungen erwies. Eine negative präoperative Diagnostik verringert die Wahrscheinlichkeit einer Infektion beträchtlich, schließt sie jedoch nicht gänzlich aus. Analog muss ein positiver präoperativer Test (z. B. mikrobiologischer Nachweis eines typischen Kontaminanten oder eine leicht erhöhte Zellzahl) immer mit der intraoperativen Diagnostik korreliert werden, um eine Überdiagnose $z u$ vermeiden.

\section{Fazit für die Praxis}

- Der Ausschluss einer periprothetischen Infektion ist von enormer Bedeutung vor einer Revisionsoperation und entscheidet in vielen Fällen über den Erfolg und die Invasivität der Operation.

- Die Diagnose „periprothetische Infektion" erfordert eine substanzielle Veränderung der therapeutischen Strategie.

- Der durch die Arbeitsgruppe entwickelte Algorithmus fasst Positionen aus der aktuellen Literatur und spezielle Expertenmeinungen zusammen.

- Da die Weiterentwicklung der Diagnostik ein dynamischer Prozess ist, erfolgt die jährlich Reevaluation. Dies ermöglicht einen transparenten diagnostischen Ansatz im Sinne einer Standard-Operation-Procedure.

- Hierdurch wird unabhängig von der Erfahrung des Untersuchers eine hohe Prozessqualität erreicht. 


\begin{tabular}{|c|c|}
\hline \multicolumn{2}{|c|}{ Abkürzungen } \\
\hline AAOS & $\begin{array}{l}\text { American Academy of Orthopa- } \\
\text { edic Surgeons }\end{array}$ \\
\hline$A M P$ & Antimikrobielle Peptide \\
\hline$B S G$ & Blutsenkungsgeschwindigkeit \\
\hline CoR & Class of Recommendation \\
\hline$C R P$ & C-reaktives Protein \\
\hline$E B J I S$ & $\begin{array}{l}\text { European Bone and Joint Infection } \\
\text { Society }\end{array}$ \\
\hline ELISA & $\begin{array}{l}\text { Enzyme-linked Immunosorbent } \\
\text { Assay }\end{array}$ \\
\hline$E S R$ & Erythrozytensedimentationsrate \\
\hline H-TEP & Hüfttotalendoprothese \\
\hline IDSA & $\begin{array}{l}\text { Infectious Diseases Society of } \\
\text { America }\end{array}$ \\
\hline ISO & $\begin{array}{l}\text { International Organization for } \\
\text { Standardisation }\end{array}$ \\
\hline ITU & $\begin{array}{l}\text { International Telecommunication } \\
\text { Union }\end{array}$ \\
\hline K-TEP & Knietotalendoprothese \\
\hline LOE & Level of Evidence \\
\hline MSIS & Musculoskeletal Infection Society \\
\hline$P C R$ & Polymerase-Kettenreaktion \\
\hline PPI & Periprothetische Infektion \\
\hline$R O C$ & $\begin{array}{l}\text { „Receiver-operating characteris- } \\
\text { tics" }\end{array}$ \\
\hline SIRS & $\begin{array}{l}\text { Systemisches inflammatorisches } \\
\text { Response-Syndrom }\end{array}$ \\
\hline SLIM & $\begin{array}{l}\text { "Synovia-like interface membra- } \\
\text { ne" }^{\prime \prime}\end{array}$ \\
\hline SOFA & $\begin{array}{l}\text { Sequential Organ Failure Assess- } \\
\text { ment }\end{array}$ \\
\hline
\end{tabular}

\section{Korrespondenzadresse}

\section{PD. Dr. H. Mühlhofer}

Klinik und Poliklinik für Orthopädie und Sportorthopädie, Klinikum rechts der Isar, Technische Universität München Ismaninger Str. 22, 81675 München, Deutschland

heinrich.muehlhofer@tum.de

Funding. Open Access funding provided by Projekt DEAL.

\section{Einhaltung ethischer Richtlinien}

Interessenkonflikt. A. Zahar: Fa. Zimmer Biomet Referent auf Honorarbasis, Fa. Waldemar Link \& Co KG - Referent auf Honorarbasis, Fa. Heraeus Medical Referent auf Honorarbasis. C. Perka: keine in Bezug auf diese Arbeit (Consultant für Zimmer/Smith\&Nephew, Link und DePuy/Synthes). L. Frommelt: Fa. Zimmer, Referent auf Honorarbasis. H. Mühlhofer, N. Renz, M. Lüdemann, M. Rudert, R. Hube, R. Ascherl und R. von Eisenhart-Rothe geben an, dass kein Interessenkonflikt besteht.

Es wurden keine Studien an Tier oder Mensch durchgeführt.

Open Access. Dieser Artikel wird unter der Creative Commons Namensnennung 4.0 International Lizenz veröffentlicht, welche die Nutzung, Vervielfältigung, Bearbeitung, Verbreitung und Wiedergabe in jeglichem Medium und Format erlaubt, sofern Sie den/die ursprünglichen Autor(en) und die Quelle ordnungsgemäß nennen, einen Link zur Creative Commons Lizenz beifügen und angeben, ob Änderungen vorgenommen wurden.

Die in diesem Artikel enthaltenen Bilder und sonstiges Drittmaterial unterliegen ebenfalls der genannten Creative Commons Lizenz, sofern sich aus der Abbildungslegende nichts anderes ergibt. Sofern das betreffende Material nicht unter der genannten Creative Commons Lizenz steht und die betreffende Handlung nicht nach gesetzlichen Vorschriften erlaubt ist, ist für die oben aufgeführten Weiterverwendungen des $\mathrm{Ma}$ terials die Einwilligung des jeweiligen Rechteinhabers einzuholen.

Weitere Details zur Lizenz entnehmen Sie bitte der Lizenzinformation auf http://creativecommons.org/ licenses/by/4.0/deed.de.

\section{Literatur}

1. Ali F, Wilkinson JM, Cooper JR, Kerry RM, Hamer AJ, Norman P, Stockley I (2006) Accuracy of Joint Aspiration for the Preoperative Diagnosis of Infection in Total Hip Arthroplasty. J Artrhoplast 21:221-226

2. Atkins BL, Athanasou N, Deeks JJ et al (1998) Prospective evaluation of criteria for microbiological diagnosis of prosthetic-joint infection at revision arthroplasty. The OSIRIS Collaborative Study Group. J Clin Microbiol 36:2932-2939

3. Banit DM, Kaufer H, Hartford JM (2002) Intraoperative frozen section analysis in revision total joint arthroplasty. Clin Orthop Relat Res 401:230-238

4. Barrack RL, Harris WH (1993) The value of aspiration of the hip joint before revision total hip arthroplasty. J Bone Joint Surg Am 75:66-76

5. Barrack RL, Jennings RW, Wolfe MW, Bertot AJ (1997) The Coventry Award. The value of preoperative aspiration before total knee revision. Clin Orthop Relat Res 345:8-16

6. Berbari E, Mabry T, Tsaras G et al (2010) Inflammatory blood laboratory levels as markers of prosthetic joint infection: a systematic review and meta-analysis.JBone Joint Surg Am 92:2102-2109. https://doi.org/10.2106/JBJS.1.01199

7. Berbari EF, Hanssen AD, Duffy MC, Steckelberg JM, Ilstrup DM, Harmsen WS et al (1998) Risk factors for prosthetic joint infection: case-control study. Clin Infect Dis 27:1247-1254

8. Berbari EF, Osmon DR, Lahr B, Eckel-Passow JE, Tsaras G, Hanssen AD et al (2012) The mayo prosthetic joint infection risk score: implication for surgical site infection reporting and risk stratification. Infect Control Hosp Epidemiol 33:774-781

9. Bogut A, Niedźwiadek J, Kozioł-Montewka Met al (2014) Sonication as a diagnostic approach used to investigate the infectious etiology of prosthetic hip joint loosening. Pol J Microbiol 63:299-306

10. Bongartz T, Halligan CS, Osmon DR, Reinalda MS, Bamlet WR, Crowson CS et al (2008) Incidence and risk factors of prosthetic joint infection after total hip or knee replacement in patients with rheumatoidarthritis. Arthritis Rheum 59:1713-1720

11. BottnerF, WegnerA, Winkelmann W, BeckerK, Erren M, Götze C (2007) Interleukin-6, procalcitonin and TNF-alpha: markers of peri-prosthetic infection following total joint replacement.J Bone Joint Surg Br 89:94-99

12. Breitenseher MJ, Mayerhöfer M, Gottsauner-Wolf F, Krestan C, Toma CD, Imhof H (2002) Diagnostic imaging in hip prostheses. Radiologe Jun:474-479

13. Coelho-Prabhu N, Oxentenko AS, Osmon DR, Baron TH, Hanssen AD, Wilson WR et al (2013) Increased risk of prosthetic joint infection associated with esophago-gastro-duodenoscopy with biopsy. SORT $84: 82-86$

14. Deirmengian C, Hallab N, Tarabishy A et al (2010) Synovial fluid biomarkers for periprosthetic infection. Clin Orthop Relat Res 468:2017-2023. https://doi.org/10.1007/s11999-010-1298-4

15. Deirmengian C, Kardos K, Kilmartin P et al (2014) Combinedmeasurement of synovial fluid-defensin and C-reactive protein levels: highly accurate for diagnosing periprosthetic joint infection. J Bone Joint Surg Am 96:1439-1445. https://doi.org/10. 2106/JBJS.M.01316

16. Deirmengian C, Kardos K, Kilmartin P et al (2014) Diagnosing periprosthetic joint infection: has the era of the biomarker arrived? Clin Orthop Relat Res 472:3254-3262. https://doi.org/10.1007/s11999014-3543-8

17. Della Valle CJ, Sporer SM, Jacobs JJ, Berger RA, Rosenberg AG, Paprosky WG (2007) Preoperative testing for sepsis before revision total knee arthroplasty. J Arthroplast 22:90-93

18. Dellinger RP, Levy MM, Rhodes A et al (2013) Surviving sepsis campaign: international guidelines for management of severe sepsis and septic shock: 2012. Crit Care Med 41(2):580-637

19. Dinneen A, Guyot A, Clements J (2013) Synovial fluid white cell and differential count in the diagnosis or exclusion of prosthetic joint infection. Bone Joint J 95:554-557

20. Dowsey MM, Choong PFM (2008) Obese diabetic patients are at substantial risk for deep infection after primary TKA. Clin Orthop Relat Res 467:1577-1581

21. Dowsey MM, Choong PFM (2008) Obesity is a major risk factor for prosthetic infection after primary hip arthroplasty. Clin Orthop Relat Res 466:153-158

22. Fink B, Gebhard A, Fuerst M et al (2013) High diagnostic value of synovial biopsy in periprosthetic joint infection of the hip. Clin Orthop Relat Res 471:956-964. https://doi.org/10. 1007/s11999-012-2474-5

23. Fink B, Makowiak C, Fuerst M et al (2008) The value of synovial biopsy, joint aspiration and C-reactive protein in the diagnosis of late periprosthetic infection of total knee replacements. 
J Bone Joint Surg Br 90:874-878. https://doi.org/ 10.1302/0301-620X.90B7.20417

24. Fink B, Makowiak C, Fuerst M, Berger I, Schäfer $P$, Frommelt $L$ (2008) The value of synovial biopsy, joint aspiration and C-reactive protein in the diagnosis of late peri-prosthetic infection of total knee replacements. J Bone Joint Surg $\mathrm{Br}$ 90:874-878

25. Francés Borrego A, Martínez FM, Cebrian Parra JL, Grañeda DS, Crespo RG, López-Durán Stern $L$ (2006) Diagnosis of infection in hip and knee revision surgery: intraoperative frozen section analysis. Int OrthopSICO 31:33-37

26. Ghanem E, Parvizi J, Burnett RSJ, Sharkey PF, Keshavarzi N, Aggarwal A et al (2008) Cell count and differential of aspirated fluid in the diagnosis of infection at the site of total knee arthroplasty. J Bone Joint Surg 90:1637-1643

27. Greidanus NV, Masri BA, Garbuz DS, Wilson SD, McAlinden MG, Xu Metal (2007) Use of erythrocyte sedimentation rate and C-reactive protein level to diagnose infection before revision total knee arthroplasty. A prospective evaluation. J Bone Joint Surg Am 89:1409-1416

28. Guyatt G, Oxman AD, Akl EA et al (2011) GRADE guidelines: 1. Introduction-GRADE evidence profiles and summary of findings tables. J Clin Epidemiol 64:383-394. https://doi.org/10.1016/j. jclinepi.2010.04.026

29. Guyatt GH, Oxman AD, Kunz R et al (2011) GRADE guidelines: 2 . Framing the question and deciding on important outcomes. J Clin Epidemiol 64:395-400. https://doi.org/10.1016/j.jclinepi. 2010.09.012

30. Guyatt GH, Oxman AD, Kunz R et al (2011) GRADE guidelines 6 . Rating the quality of evidenceimprecision. J Clin Epidemiol 64:1283-1293. https://doi.org/10.1016/j.jclinepi.2011.01.012

31. Guyatt GH, Oxman AD, Kunz R et al (2011) GRADE guidelines: 8 . Rating the quality of evidenceindirectness. J Clin Epidemiol 64:1303-1310. https://doi.org/10.1016/j.jclinepi.2011.04.014

32. Guyatt GH, Oxman AD, Montori V et al (2011) GRADE guidelines: 5 . Rating the quality of evidence-publication bias. J Clin Epidemiol 64:1277-1282. https://doi.org/10.1016/j.jclinepi. 2011.01.011

33. Guyatt GH, Oxman AD, Sultan S et al (2011) GRADE guidelines: 9. Rating up the quality of evidence. JClin Epidemiol 64:1311-1316. https://doi.org/10. 1016/j.jclinepi.2011.06.004

34. Guyatt GH, Oxman AD, Vist G et al (2011) GRADE guidelines: 4 . Rating the quality of evidencestudy limitations (risk of bias). J Clin Epidemiol 64:407-415. https://doi.org/10.1016/j.jclinepi. 2010.07.017

35. Huotari K, Lyytikäinen O, Seitsalo S (2007) Patient outcomes after simultaneous bilateral total hip and knee joint replacements. J Hosp Infect 65:219-25

36. Jämsen E(2009) Riskfactors for infection after knee arthroplasty. J Bone Joint Surg Am 91:38

37. Kamme C, Lindberg L (1981) Aerobic and anaerobic bacteria in deep infections after total hip arthroplasty: differential diagnosis between infectious and non-infectious loosening. Clin Orthop Relat Res 154:201-207

38. Kasparek MF, Kasparek M, Boettner F, Faschingbauer M, Hahne J, Dominkus M et al (2016) Intraoperative Diagnosis of Periprosthetic Joint Infection Using a Novel Alpha-Defensin Lateral Flow Assay. J Arthroplasty. https://doi.org/10. 1016/j.arth.2016.05.033

39. Khalil PN, Kleespies A, Angele MK et al (2010) The formal requirements of algorithms and their implications in clinical medicine and quality management. Langenbecks Arch Surg 396:31-40. https://doi.org/10.1007/s00423-010-0713-3

40. Krenn V, Morawietz L, Kienapfel H et al (2013) Revised consensus classification. Z Rheumatol 72:383-392. https://doi.org/10.1007/s00393012-1099-0

41. Kurtz S (2007) Projections of primary and revision hip and knee arthroplasty in the United States from 2005 to 2030. JBone Joint Surg Am 89:780. https:// doi.org/10.2106/JBJS.F.00222

42. Kurtz SM, Ong KL, Lau E, Bozic KJ, Berry D, Parvizi $J(2009)$ Prosthetic joint infection risk after TKA in the medicare population. Clin Orthop Relat Res 468:52-56

43. Lachiewicz PF, Rogers GD, Thomason HC (1996) Aspiration of the hip joint before revision total hip arthroplasty. Clinical and laboratory factors influencing attainment of a positive culture. J Bone Joint Surg Am 78:749-754

44. Llewelyn M, Cohen J, International Sepsis Forum (2001) Diagnosis of infection in sepsis. Intensive Care Med 27(Suppl 1):10-32

45. Lübbeke A, Stern R, Garavaglia G, Zurcher L, Hoffmeyer P (2007) Differences in outcomes of obese women and men undergoing primary tota hip arthroplasty. Arthritis Rheum 57:327-334

46. Luessenhop CP, Higgins LD, Brause BD, Ranawat CS (1996) Multiple prosthetic infections after toal joint arthroplasty. Risk factor analysis. J Arthroplasty 11:862-868

47. Malhotra R, Morgan D (2004) Role of core biopsy in diagnosing infection before revision hip arthroplasty. J Arthroplasty. https://doi.org/10. 1016/S0883-5403

48. Malinzak RA, Ritter MA, Berend ME, Meding JB, Olberding EM, Davis KE (2009) Morbidly obese, diabetic, younger, and unilateral joint arthroplasty patients have elevated total joint arthroplasty infection rates. J Arthroplast 24:84-88

49. Marin M, Garcia-Lechuz JM, Alonso $P$ et al (2012) Role of universal 16S rRNA gene PCR and sequencing in diagnosis of prosthetic joint infection. J Clin Microbiol 50:583-589. https://doi. org/10.1128/JCM.00170-11

50. McArthur BA, Abdel MP, Taunton MJ et al (2015) Seronegative infections in hip and knee arthroplasty: periprosthetic infections with normal erythrocyte sedimentation rate and C-reactive protein level. Bone Joint J 97-B:939-944. https:// doi.org/10.1302/0301-620X.97B7.35500

51. Moher D, Liberati A, Tetzlaff J et al (2009) Preferred reporting items for systematic reviews and meta-analyses: the PRISMA statement. PLoS Med 6:e1000097.https://doi.org/10.1371/journal. pmed.1000097

52. Mraovic B, Suh D, Jacovides C, Parvizi J (2011) Perioperative hyperglycemia and postoperative infection after lower limb arthroplasty. J Diabetes Sci Technol 5:412-418

53. Mulcahy DM, Fenelon GC, Mclnerney DP (1996) Aspiration arthrography of the hip joint. Its uses and limitations in revision hip surgery.J Arthroplast 11:64-68

54. Murdoch DR, Roberts SA, Fowler VG Jr, Shah MA, Taylor SL, Morris AJ et al (2001) Infection of orthopedic prostheses after Staphylococcus aureus bacteremia. Clin Infect Dis 32:647-649

55. Murray RP, Bourne MH, Fitzgerald RH (1991) Metachronous infections in patients who have had more than one total joint arthroplasty. J Bone Joint Surg Am 73:1469-1474

56. Namba RS, Inacio M, Paxton EW (2012) Risk factors associated with surgical site infection in 30,491 primary total hip replacements. J Bone Joint Surg Br94:1330-1338

57. Namba RS, Inacio MCS, Paxton EW (2013) Risk factors associated with deepsurgical siteinfections after primary total knee arthroplasty. J Bone Joint Surg Am 95:775-778

58. Osmon DR, Berbari EF, Berendt AR et al (2013) Diagnosis and management of prosthetic joint infection: clinical practice guidelines by the Infectious Diseases Society of America. Clin Infect Dis 56:e1-e25. https://doi.org/10.1093/cid/cis803

59. Oussedik S, Gould K, Stockley I (2012) Defining peri-prosthetic infection. J Bone Joint Surg Br. https://doi.org/10.1302/0301-620X.94B11

60. Parvizi J, Della Valle CJ (2010) AAOS clinical practice guideline: diagnosis and treatment of periprosthetic joint infections of the hip and knee. JAm Acad Orthop Surg 18:771-772

61. Parvizi J, Gehrke T, Chen AF (2013) Proceedings of the International Consensus on Periprosthetic Joint Infection. Bone Joint J 95-B:1450-1452

62. Peel TN, Dowsey MM, Daffy JR, Stanley PA, Choong PFM, Buising KL (2011) Risk factors for prosthetic hip and knee infections according to arthroplasty site. J Hosp Infect 79:129-133

63. Peersman G, Laskin R, Davis J, Peterson M (2001) Infection in total knee replacement: a retrospective review of 6489 total knee replacements. Clin Orthop Relat Res 392:15-23

64. Pohlig F, Mühlhofer HML, Lenze U et al (2017) Diagnostic accuracy of arthroscopic biopsy in periprosthetic infections of the hip. Eur J Med Res 22:1-7. https://doi.org/10.1186/s40001-0170246-0

65. Portillo ME, Salvadó M, Alier A, Sorli L, Martínez S, Horcajada JP et al (2013) Prosthesis failure within 2 years of implantation is highly predictive of infection. Clin Orthop Relat Res 471:3672-3678

66. Pulido L, Ghanem E, Joshi A, Purtill JJ, Parvizi J (2008) Periprostheticjoint infection: the incidence, timing, and predisposing factors. Clin Orthop Relat Res 466:1710-1715

67. Rakow A, Perka C, Trampuz A, Renz N (2019) Origin and characteristics of haematogenous periprosthetic joint infection. Clin Microbiol Infect 25:845-850. https://doi.org/10.1016/j.cmi.2018. 10.010

68. Renz N, Yermak K, Perka C, Trampuz A (2018) Alpha defensin lateral flow test for diagnosis of periprosthetic joint infection. J Bone Joint Surg Am 100:742-750. https://doi.org/10.2106/JBJS. 17.01005

69. Sackman H (1974) Delphi Assessment: expert opinion, forecasting and group process, S 1-130

70. Saleh KJ, Clark CR, Sharkey PF et al (2003) Modes of failure and preoperative evaluation. JBone Joint Surg Am 85-A(Suppl 1):S21-S25

71. Savarino L, Baldini N, Tarabusi C, Pellacani A, Giunti A (2004) Diagnosis of infection after total hip replacement. JBiomed Mater Res 70:139-145

72. Schäfer P, Fink B, Sandow D et al (2008) Prolonged bacterial culture to identify late periprosthetic joint infection: a promising strategy. Clin Infect Dis 47:1403-1409. https://doi.org/10.1086/592973

73. Schinsky MF (2008) Perioperative testing for joint infection in patients undergoing revision total hip arthroplasty. JBone Joint Surg Am 90:1869. https:// doi.org/10.2106/JBJS.G.01255

74. Seymour CW, Liu VX, Iwashyna TJ et al (2016) Assessment of clinical criteria for sepsis: for the third international consensus definitions for sepsis and septic shock (sepsis-3). JAMA 315:762-774. https://doi.org/10.1001/jama.2016.0288 
75. Småbrekke A, Espehaug B, Havelin LI, Furnes 0 (2004) Operating time and survival of primary total hip replacements: an analysis of 31,745 primary cemented and uncemented total hip replacements from local hospitals reported to the Norwegian Arthroplasty Register 1987-2001. Acta Orthop Scand 75:524-532

76. Society TWCBTMI (2011) New Definition for Periprosthetic Joint Infection. J Arthroplast 26:1136-1138. https://doi.org/10.1016/j.arth. 2011.09.026

77. Sousa R, Muñoz-Mahamud E, Quayle J, Dias da Costa L, Casals C, Scott P et al (2014) Is asymptomatic bacteriuria a risk factor for prosthetic joint infection? Clin Infect Dis 59:41-47

78. Spangehl MJ, Masri BA, O'connell JX, Duncan CP (1999) Prospective analysis of preoperative and intraoperative investigations for the diagnosis of infection at the sites of two hundred and two revision total hip arthroplasties. J Bone Joint Surg Am 81:672-683

79. Teller RE, Christie MJ, Martin W, Nance EP, Haas DW (2009) Sequential indiumlabeled leukocyte and bone scans to diagnose prosthetic joint infection. Clin Orthop Relat Res 373:241-247

80. Trampuz A, Piper KE, Jacobson MJ et al (2007) Sonication of removed hip and knee prostheses for diagnosis of infection. N Engl J Med 357:654-663. https://doi.org/10.1056/NEJMoa061588

81. Urquhart DM, Hanna FS, Brennan SL et al (2010) Incidence and risk factors for deep surgical site infection after primary total hip arthroplasty: a systematic review. J Arthroplasty 25(3):1216-1222.e1. https://doi.org/10.1016/j. arth.2009.08.011

82. Uçkay I, Lübekke A, Emonet S, Tovmirzaeva L, Stern R, Ferry T et al (2009) Low incidence of haematogenous seeding to total hip and knee prostheses in patients with remote infections. Infect 59:337-345

83. Virolainen $P$, Lähteenmäki $H$, Hiltunen $A$, Sipola E, Meurman O, Nelimarkka O (2002) The reliability of diagnosis of infection during revision arthoplasties. Scand J Surg 91:178-181

84. Williams JL, Norman P, Stockley I (2004) The value of hip aspiration versus tissue biopsy in diagnosing infection before exchange hip arthroplasty surgery. J Arthroplasty. https://doi. org/10.1016/j.arth.2003.11.011

85. Wyatt MC, Beswick AD, Kunutsor SK et al (2016) The alpha-defensin immunoassay and leukocyte esterase colorimetric strip test for the diagnosis of periprosthetic infection. J Bone Joint Surg Am 98:992-1000. https://doi.org/10.2106/JBJS. 15.01142

86. Yee DKH, Chiu KY, Yan CH, Ng FY (2013) Review article: joint aspiration for diagnosis of periprostheticinfection.J Orthop Surg 21:236-240

87. ZimmerliW, TrampuzA, OchsnerPE(2004)Prosthetic-joint infections. N Engl J Med 351:1645-1654. https://doi.org/10.1056/NEJMra040181

88. Zmistowski B, Restrepo C, Huang R, Hozack WJ, Parvizi J (2012) Periprosthetic joint infection diagnosis. J Arthroplast 27:1589-1593

89. Zmistowski B, Karam JA, Durinka JB et al (2013) Periprosthetic joint infection increases the risk of one-year mortality. J Bone Joint Surg Am 95:2177-2184. https://doi.org/10.2106/JBJS.L. 00789

\section{Diabetischer Fuß}

\section{Wie Telemedizin, zertifizierte Teams und neueste Behandlungsmetho-} den Gliedmaßen retten können

Das diabetische Fußsyndrom (DFS) ist nach wie vor eine der häufigsten Folgeund Begleiterkrankungen bei Diabetes Typ 1 und Typ 2. Hierzulande werden jährlich bis zu 50.000 Amputationen aufgrund eines DFS durchgeführt. Durch flächendeckende, gezielte Maßnahmen könnte diese Zahl deutlich reduziert werden, konstatiert die Arbeitsgemeinschaft „Diabetischer Fuß" der Deutschen Diabetes Gesellschaft (DDG).

Muss ein Zeh, ein Teil des Fußes oder schließlich gar ein ganzes Bein aufgrund eines diabetischen Fußsyndroms amputiert werden, stellt dies für behandelnde Ärzte eine schwere Entscheidung mit unumkehrbaren Folgen für die Mobilität und die Lebensqualität betroffener Patienten dar. „Geeignete Präventionsmaßnahmen und interdisziplinäre ärztliche Zusammenarbeit kann Betroffenen einen solchen Weg ersparen", erklärt Dr. med. Michael Eckhard, Sprecher der AG „Diabetischer Fuß" der DDG. Dass diesbezüglich noch viel zu tun ist, zeigen die Zahlen: Noch immer gehen etwa zwei Drittel aller jährlichen Amputationen in Deutschland auf das diabetische Fußsyndrom zurück - rund die Hälfte wäre vermeidbar.

Nationale wie internationale Studienergebnisse zeigen, dass es beim DFS auf eine frühzeitige Zuweisung des Patienten an eine spezialisierte Behandlungseinrichtung ankommt. „In zertifizierten Zentren liegt die Rate der Major-Amputationen nur bei etwa $3 \%$, während sie in der Regelversorgung dagegen noch bei über $10 \%$ liegt", betont Eckhard. Er fordert daher: „Alle Menschen mit DFS müssen an ein multidisziplinäres Fußbehandlungsteam verwiesen werden und zwar ohne Verzögerung."

Seit 15 Jahren setzt sich die AG im Rahmen eines strukturierten Zertifizierungsverfahrens von Behandlungseinrichtungen für den Erhalt amputationsbedrohter Extremitäten bei Menschen mit Diabetes mellitus ein. Diese Arbeit hat bereits Früchte getragen: So hat der Gemeinsame Bundesausschuss (G-BA) dieses Jahr beschlossen, dass gesetzlich krankenversicherte Patientinnen und Patienten mit einem DFS vor einer geplanten Amputation einen Rechtsanspruch auf Einholung einer unabhängigen ärztlichen Zweitmeinung haben. Hierfür ist die AG derzeit an einem Stellungnahmeverfahren zu einer vom In- stitut für Qualität und Wirtschaftlichkeit im Gesundheitswesen (IQWiG) erstellten Entscheidungshilfe für Amputationen beim DFS beteiligt.

„Darüber hinaus haben wir ein im klinischen Alltag einsetzbares Tool auf telemedizinischer Basis entwickelt, welches eine zeitlich und örtlich unabhängige qualifizierte Zweitmeinung ermöglicht", führt Professor Dr. med. Ralf Lobmann aus, der dieses Projekt federführend begleitet. Es soll dazu beitragen, dem Patienten eine zeitgerechte Entscheidungshilfe an die Hand zu geben, ohne die Versorgungseinrichtung verlassen zu müssen. Denn: „Steht eine große, sogenannte Major-Amputation an, ist die Situation immer dringlich und erfordert eine Entscheidung binnen maximal 36 Stunden." Das sei unter derzeitigen Bedingungen kaum realisierbar. Anknüpfend an diesem Modell entwickelt die DDG in Zusammenarbeit mit dem Berufsverband Deutscher Internisten (BDI) und weiterer Kooperationspartner eine Plattform für ein telemedizinisches Fußkonsil. Kürzlich ist auch der Fuß-Pass der DDG erschienen: Er soll durch gezielte Patientenaufklärung das Zweitmeinungsverfahren bekannt machen und zur Senkung der hohen Amputationsrate beitragen.

Literatur:

Gemeinsamer Bundesausschuss (2020) Richtlinie zum Zweitmeinungsverfahren: Aufnahme des Eingriffs Amputationen beim Diabetischen Fußsyndrom sowie weitere Änderung der Richtlinie: https://www.gba.de/beschluesse/4266/

Quelle: Deutsche Diabetes Gesellschaft (DDG), www.deutsche-diabetesgesellschaft.de 
Hier steht eine Anzeige.

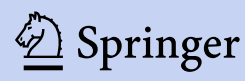

